\title{
Automorphic vector bundles on connected Shimura varieties*
}

\section{J.S. Milne}

Mathematics Department, University of Michigan, Ann Arbor, MI 48109, USA

Introduction

0 . Review of terminology concerning Shimura varieties 93

1. The Taniyama group, the period torsor, and conjugates of Shimura varieties 95

2. The compact dual symmetric Hermitian space and its conjugates 101

3. The principal bundle $Y^{0}(G, X)$; statement of the first main theorem 105

4. Automorphic vector bundles $\quad 112$

$\begin{array}{ll}\text { 5. Conjugates of automorphic vector bundles } & 115\end{array}$

6. Proof of Theorem 3.10 for the symplectic group $\quad 118$

7. Proof of Theorem 3.10 for connected Shimura varieties of abelian type 121

$\begin{array}{ll}\text { 8. First completion of the proof of Theorem } 3.10 & 123\end{array}$

9. Second completion of the proof of Theorem $3.10 \quad 124$

Appendix: Pairs defining connected and nonconnected Shimura varieties $\quad 126$

$\begin{array}{lr}\text { Bibliography } & 128\end{array}$

\section{Introduction}

A connected Shimura variety $S^{0}(G, X)$ is defined by a semisimple group $G$ over $\mathbb{Q}$ and a symmetric Hermitian domain $X$. For any automorphism $\tau$ of $\mathbb{C}$ (as an abstract field), it is known that the conjugate $\tau S^{0}(G, X)$ of $S^{0}(G, X)$ has a canonical realization as a connected Shimura variety $S^{0}\left(G^{\prime}, X^{\prime}\right)$, and that the pair $\left(G^{\prime}, X^{\prime}\right)$ defining the second Shimura variety can be constructed from the first pair by using the Taniyama group. In more down-to-earth terms, we can say that with an automorphic function $f$ on $X$ and a special point $x$ of $X$, it is possible to associate a new automorphic function ${ }^{t, x} f$ on a different domain $X^{\prime}$; the associated $f \mapsto^{\mathrm{r}, x} f$ commutes with the Hecke operators, and $\tau(f(x))$ $={ }^{r, x} f\left(x^{\prime}\right)$ for an explicitly defined special point $x^{\prime}$ on $X^{\prime}$. (A proof of this result for most connected Shimura varieties can be found in Milne and Shih (1982b) and for the remaining varieties in Milne (1983); see also Borovoi (1983/4). It is the analogue for connected Shimura varieties of a conjecture of Langlands (1979).)

\footnotetext{
* Parts of this work have " while the author was at th:
} supported by the National Science Foundation; it was completed smatical Sciences Research Institute, Berkeley 
The purpose of the present paper is to extend these statements to holomorphic automorphic forms. Such forms of a fixed type are sections of a vector bundle over a connected Shimura variety. The vector bundles for whose sections our results hold arise from equivariant vector bundles on the compact dual of $X$; we call them automorphic vector bundles (the importance of these vector bundles has been emphasized by P. Deligne; the name was suggested to me by $M$. Harris). Holomorphic automorphic forms in the classical sense corresponding to automorphy factors for the full group arise as sections of automorphic vector bundles. We show in $\S 5$ that the conjugate $\tau \mathscr{V}$ of an automorphic vector bundle can be canonically realized as an automorphic vector bundle on $S^{0}\left(G^{\prime}, X^{\prime}\right)$. More precisely, we show (Theorem 5.2) that there is an automorphic vector bundle $\mathscr{V}^{\prime}$ on $S^{0}\left(G^{\prime}, X^{\prime}\right)$ and a canonical isomorphism $\tau \mathscr{V} \stackrel{\approx}{\longrightarrow} \mathscr{W}^{\prime}$ lifting the isomorphism $\tau S^{0}(G, X) \stackrel{\approx}{\longrightarrow} S^{0}\left(G^{\prime}, X^{\prime}\right)$ and commuting with the Hecke operators; the data defining $\mathscr{V}^{\prime}$ is constructed from the that defining $\mathscr{V}$ by using the Taniyama group and the period torsor. In more downto-earth terms, we can say that with an automorphic form $f$ and a special point $x$ of $X$, it is possible to associate a new automorphic form ${ }^{\tau, x} f$ on the domain $X^{\prime}$; the association $f \mapsto^{\tau, x} f$ commutes with the Hecke operators, and $\tau(f(x))$ can often be related to ${ }^{t, x} f\left(x^{\prime}\right)$ where $x^{\prime}$ is the same special point of $X^{\prime}$ as above.

In a sequel to this paper, these results will be used to obtain similar results for automorphic forms on nonconnected Shimura varieties. In this way, we shall obtain an analogue for automorphic vector bundles over Shimura varieties of Langlands's conjecture on the conjugates of Shimura varieties. In particular, this will allow us (without any assumptions on the underlying Shimura variety) to define canonical models of automorphic vector bundles, and to give a definitive definition of what it means for a holomorphic automorphic form to be rational over a number field.

The theorem on automorphic vector bundles is obtained as a rather direct consequence of a theorem (again a generalization of Langlands's conjecture) concerning a certain principal bundle $Y^{0}(G, X)$ over $S^{0}(G, X)$. This theorem is stated in $\S 3$ and proved in $\S 6$ and $\S 7$ for connected Shimura varieties of abelian type. In $\S 8$ and $\S 9$ we give two methods of extending the result to all connected Shimura varieties. The first, which is the shorter, uses a statement (Borovoi (1983/4), 3.21) for which no proof is currently available; the second makes use of an idea from Harris (1985). Automorphic vector bundles are defined in $\S 4$ and their conjugates are described in $\S 5$. The first two sections contain preliminary material on the Taniyama group, the period torsor, conjugates of connected Shimura varieties, and conjugates of the compact duals of Hermitian symmetric domains.

Lacking at this point are theorems describing how automorphisms of $\mathbb{C}$ act on the Fourier-Jacobi series of automorphic forms (or even a general algebraic definition of such series) and on the Eisenstein series associated with cusp forms on boundary components. It is however possible to give precise conjectures, again in terms of the Taniyama group and the period torsor, and I hope to take up these questions in future papers. 
A discussion of the relation of these results to those of other authors will be given in the sequel to this paper. Here we mention only that the results were suggested by those of Harris $(1984,1985)$, which in turn were suggested by questions of Shimura (1980) and Deligne.

\section{Review of terminology concerning Shimura varieties}

A reductive group is always assumed to be connected. When $G$ is an algebraic group, $G^{\text {der }}$ and $G^{\text {ad }}$ are the associated derived and adjoint groups of $G$, and $Z(G)$ is the centre of $G$. The action of $G$ on itself by inner automorphisms factors through a homomorphism ad: $G^{\text {ad }} \rightarrow \operatorname{Aut}(G)$. The simply connected covering group of a semisimple group $G$ is denoted by $\widetilde{G}$.

When $G$ is an algebraic group over $\mathbb{R}, G(\mathbb{R})^{+}$is the identity component of $G(\mathbb{R})$ (for the real topology), and $G(\mathbb{R})_{+}$is the inverse image of $G^{\text {ad }}(\mathbb{R})^{+}$ in $G(\mathbb{R})$. In the case that $G$ is defined over $\mathbb{Q}$, we write $G(\mathbb{Q})^{+}$for $G(\mathbb{Q}) \cap G(\mathbb{R})^{+}$ and $G(\mathbb{Q})_{+}$for $G(\mathbb{Q}) \cap G(\mathbb{R})_{+}$. The symbol ${ }^{-}$denotes closure in $G\left(\mathbb{A}^{f}\right)$ where $\mathbb{A}^{f} \stackrel{d f}{=} \widehat{\mathbb{Z}} \otimes \mathbb{Q}$ is the ring of finite adèles. We write $\mathbb{A}$ for $\mathbb{R} \times \mathbb{A}^{f}$ and $\mathbb{A}^{\prime}$ for $\mathbb{C} \times \mathbb{A}^{f}$.

The real torus $\operatorname{Res}_{\mathbb{C} / \mathbb{R}} \mathbb{G}_{m}$ is denoted by $\mathbf{S}$; thus $\mathbf{S}(\mathbb{R})=\mathbb{C}^{\times}$and $\mathbf{S}(\mathbb{C})=\mathbb{C}^{\times} \times \mathbb{C}^{\times}$ (the projections onto the two factors correspond respectively to the identity automorphism of $\mathbb{C}$ and to complex conjugation). Associated with any homomorphism $h: \mathbb{S} \rightarrow G$, there is a weight map

$$
w_{h}: \mathbb{G}_{m} \rightarrow G, \quad r \mapsto h(r)^{-1}, \quad \text { all } r \in \mathbb{R}^{\times} \subset \mathbf{S}(\mathbb{R}),
$$

and a cocharacter

$$
\mu_{h}: \mathbb{G}_{m} \rightarrow G_{\mathbb{C}}, \quad z \mapsto h_{\mathbb{C}}(z, 1), \quad \text { all } z \in \mathbb{C}^{\times} .
$$

By a pair $(G, X)$ defining a connected Shimura variety we mean a semisimple algebraic group $G$ over $\mathbb{Q}$ and a $G^{\text {ad }}(\mathbb{R})^{+}$-conjugacy class $X$ of homomorphisms $\mathbb{S} \rightarrow G_{\mathbf{R}}^{\text {ad }}$ satisfying the conditions (2.1.1) $-(2.1 .3)$ of Deligne (1979). Then $X$ has a canonical structure of a Hermitian symmetric domain, and we write $x$ for a point of $X$ when we are regarding it in this way and $h_{x}: \mathbf{S} \rightarrow G_{\mathbf{R}}^{\text {ad }}$ and $\mu_{x}: \mathbb{G}_{m} \rightarrow G_{\mathbb{C}}^{\text {ad }}$ for the homomorphism and cocharacter associated with $x$. The connected Shimura variety $S^{0}(G, X)$ is defined to be the projective system $(\Gamma \backslash X)_{\Gamma \in \Sigma(G)}$ (or its limit), where $\Sigma(G)$ is the set of net arithmetic subgroups of $G^{\text {ad }}(\mathbb{Q})$ containing the image of a congruence subgroup in $G(\mathbb{Q})$. Each complex manifold $\Gamma \backslash X$ has a unique structure as an algebraic variety, and the morphisms in the projective system are algebraic. It is sometimes also convenient to regard $S^{0}(G, X)$ as being the projective system of varieties $(\Gamma \backslash X)$ with $\Gamma$ running over the set $\tilde{\Sigma}(G)$ of net congruence subgroups of $G(\mathbb{Q})$. We often write $S_{\Gamma}^{0}(G, X)$ (or $S_{\mathrm{K}}^{0}(G, X)$ when $\Gamma=G(\mathbb{Q}) \cap K, K$ compact an open in $G\left(\mathbb{A}^{f}\right)$ ) for the algebraic variety $\Gamma \backslash X$. When $G$ is simply connected,

$$
S^{o}(G, X)(\mathbb{C}) \stackrel{d f}{=} \lim _{\leftarrow} S_{\Gamma}^{0}(G, X)(\mathbb{C})=G(\mathbb{Q}) \backslash X \times G\left(\mathbb{A}^{f}\right) .
$$


In the last term, $q \in G(\mathbb{Q})$ acts on $(x, g) \in X \times G\left(\mathbb{A}^{f}\right)$ according to the rule:

$$
q(x, g)=(q x, q g) \text {. }
$$

For $x$ in $X, x_{\Gamma}$ denotes the image of $x$ in $\Gamma \backslash X$, and $[x]=\left(x_{\Gamma}\right)_{\Gamma \in \Sigma(G)}$ denotes its image in $S^{0}(G, X)$.

The action of $G^{\text {ad }}(\mathbb{Q})^{+}$on $S^{\circ}(G, X)$,

$$
g: \Gamma \backslash X \rightarrow \Gamma^{\prime} \backslash X, \quad x_{\Gamma} \mapsto(g x)_{\Gamma^{\prime}}, \quad \Gamma^{\prime}=\operatorname{ad}(\mathrm{g}) \Gamma
$$

extends by continuity to the completion $G^{\text {ad }}(\mathbb{Q})^{+\wedge}$ of $G^{\text {ad }}(\mathbb{Q})^{+}$for the topology defined by the subgroups in $\Sigma(G)$. The $\operatorname{map} G(\mathbb{Q})_{+} \rightarrow G^{\text {ad }}(\mathbb{Q})^{+\wedge}$ extends by continuity to the closure $G(\mathbb{Q})_{+}^{-}$of $G(\mathbb{Q})_{+}$in $G\left(\mathbb{A}^{f}\right)$, and $G^{\text {ad }}(\mathbb{Q})^{+\wedge}$ is generated by $G^{\text {ad }}(\mathbb{Q})^{+}$and the image $G(\mathbb{Q})_{+}^{-} / Z(\mathbb{Q})$ of $G(\mathbb{Q})_{+}^{-}$; more precisely,

$$
G^{\text {ad }}(\mathbb{Q})^{+\wedge}=G(\mathbb{Q})_{+* G(\mathbb{Q})_{+}}^{-a \mathrm{ad}}(\mathbb{Q})^{+}
$$

(Deligne (1979), 2.1.6.2). In fact $G^{\text {ad }}(\mathbb{Q})^{+\wedge}$ is also generated by $G^{\text {ad }}(\mathbb{Q})^{+}$and the image of $\tilde{G}\left(\mathbb{A}^{f}\right)$. In the case that $G$ is simply connected, $G(\mathbb{Q})_{+}^{-}=G\left(\mathbb{A}^{f}\right)$, and the actions of $G\left(\mathbb{A}^{f}\right)$ and $G^{\text {ad }}(\mathbb{Q})^{+}$on $S^{0}(G, X)$ are given by

$$
\begin{aligned}
& a[x, g]=\left[x, g a^{-1}\right], \quad x \in X, \quad a, g \in G\left(\mathbb{A}^{f}\right) ; \\
& q[x, g]=[g x, \operatorname{ad}(q) g], \quad x \in X, \quad q \in G^{a d}(\mathbb{Q})^{+}, \quad g \in G\left(\mathbb{A}^{f}\right) .
\end{aligned}
$$

We write (g) for the automorphism of $S^{\circ}(G, X)$ defined by $g \in G^{\text {ad }}(\mathbb{Q})^{+\wedge}$.

By a morphism $f:(G, X) \rightarrow\left(G^{\prime}, X^{\prime}\right)$ of pairs defining connected Shimura varieties we mean a homomorphism $f: G \rightarrow G^{\prime}$ of algebraic groups over $\mathbb{Q}$ carrying the conjugacy class $X$ into $X^{\prime}$. The map $h \mapsto \operatorname{ad}(f) \circ h: X \rightarrow X^{\prime}$ automatically sends special points of $X$ to special points of $X^{\prime}$. Such an $f$ defines a morphism $S^{0}(f): S^{0}(G, X) \rightarrow S^{0}\left(G^{\prime}, X^{\prime}\right)$ of connected Shimura varieties taking the action of $g \in G\left(\mathbb{A}^{f}\right)$ into that of $f(g) \in G^{\prime}\left(\mathbb{A}^{f}\right)$. We say that $f$ is an embedding if $f: G \rightarrow G^{\prime}$ is injective. In this case $S^{0}(f)$ is a projective system of closed immersions (see Deligne (1971), 1.15).

By a pair $\left(G_{1}, X_{1}\right)$ defining a Shimura variety we mean a reductive group $G_{1}$ over $\mathbb{Q}$ and a $G_{1}(\mathbb{R})$-conjugacy class $X_{1}$ of homomorphisms $\mathbf{S} \rightarrow G_{1 \mathbf{R}}$ satisfying the conditions (2.1.1)-(2.1.3) of (Deligne (1979)). For such a pair $\left(G_{1}, X_{1}\right)$, a connected component $X_{1}^{+}$of $X_{1}$ can be identified with a $G_{1}^{\text {ad }}(\mathbb{R})^{+}$-conjugacy $d f$

class of maps $\mathbf{S} \rightarrow G_{1 \mathrm{R}}^{\text {ad }}$, and $(G, X)=\left(G_{1}^{\text {der }}, X_{1}^{+}\right)$is a pair defining a connected Shimura variety; in this situation, we write $(G, X)=\left(G_{1}, X_{1}\right)^{+}$. The connected component of $S\left(G_{1}, X_{1}\right)$ containing the image of $X_{1}^{+}$can be identified with $S^{0}(G, X)$. The action of $G_{1}\left(\mathbb{A}^{f}\right)$ on $S\left(G_{1}, X_{1}\right)$ factors through $G_{1}\left(\mathbb{A}^{f}\right) / Z_{1}(\mathbb{Q})^{-}$, where $Z_{1}$ is the centre of $G_{1}$, and the stabilizer of $S^{0}(G, X)$ in $S\left(G_{1}, X_{1}\right)$ is $G_{1}(\mathbb{Q})_{+}^{-} / Z_{1}(\mathbb{Q})^{-}$(here - denotes closure in $G_{1}\left(\mathbb{A}^{f}\right)$ ). If we assume that $H^{1}\left(\mathbb{Q}, Z_{1}\right)=0$, then $G^{\text {ad }}(\mathbb{Q})=G_{1}(\mathbb{Q}) / Z_{1}(\mathbb{Q}) \subset G_{1}\left(\mathbb{A}^{f}\right) / Z_{1}(\mathbb{Q})^{-}$, and the stabilizer of $S^{0}(G, X)$ in $G_{1}\left(\mathbb{A}^{f}\right) / Z_{1}(\mathbb{Q})^{-}$is the closure of $G^{\text {ad }}(\mathbb{Q})^{+}$in $G_{1}\left(\mathbb{A}^{f}\right) / Z_{1}(\mathbb{Q})^{-}$. This closure can be identified with $G^{\text {ad }}(\mathbb{Q})^{+\wedge}$, and its action on $S^{0}(G, X)$, when converted into a left action, agrees with that defined above [ibid. 2.1.16]. 
All vector spaces and vector bundles are of finite dimension. The category of representations of an algebraic group $G$ on $k$-vector spaces is denoted by $\operatorname{Rep}_{k}(G)$.

Motives are always meant in the sense of (absolute) Hodge cycles (see (Deligne and Milne (1982), §6)).

We rarely distinguish a vector bundle from its associated locally free sheaf of sections. By a variation of real Hodge structures on a complex manifold $X$, we mean a local system of real vector spaces $\mathbf{V}$ on $X$ together with, at each point $x$ of $X$, a real Hodge structure on the fibre $\mathbf{V}_{x}$ at $x$; these Hodge structures are required to vary continuously in $x$, and the associated Hodge filtrations $F^{*}$ on the fibres of $\mathcal{O}_{X} \otimes \mathbf{V}$ are required to vary holomorphically and satisfy the axiom of transversality: $\nabla F^{p} \subset \Omega_{X}^{1} \otimes F^{p-1}$ (Deligne 1979), 1.1.7). To define a variation of rational Hodge structures replace "real" with "rational" in the preceding definition.

The algebraic closure of $\mathbb{Q}$ in $\mathbb{C}$ is denoted by $\mathbb{Q}$. When necessary, we denote the inclusion $\mathbb{Q} \hookrightarrow \mathbb{C}$ by $\imath$. We often use $=$ to denote a canonical isomorphism. The equivalence class containing ${ }^{*}$ is often written $\left[{ }^{*}\right]$.

\section{The Taniyama group, the period torsor, and conjugates of Shimura varieties}

Recall (Milne and Shih 1982a) that the Serre group is a pair $\left(\Im, h_{\text {can }}\right)$ consisting of a proalgebraic torus $\mathbb{S}$ over $\mathbb{Q}$ and a homomorphism $h_{\text {can }}: \mathbf{S} \rightarrow \mathbb{S}_{\mathbb{R}}$ whose weight is defined over $\mathbb{Q}$. The pair is universal in the following sense: for any torus $T$ over $\mathbb{Q}$ and homomorphism $h: \mathbf{S} \rightarrow T_{\mathbb{R}}$ whose cocharacter $\mu$ is defined over a $C M$ field and whose weight $w$ is defined over $\mathbb{Q}$, there is a unique Q-rational homomorphism $\rho: \mathbb{S} \rightarrow T$ such that $\rho_{\mathbf{R}}{ }^{\circ} h_{\text {can }}=h$.

The Taniyama group [ibid. §3] is an extension

$$
1 \rightarrow \mathfrak{S} \rightarrow \mathfrak{I} \stackrel{\pi}{\longrightarrow} \operatorname{Gal}(\Phi / \mathbb{Q}) \rightarrow 1
$$

of pro-algebraic groups together with a continuous section sp: $\mathrm{Gal}(\overline{\mathbb{Q}} / \mathbb{Q}) \rightarrow \mathfrak{I}\left(\mathbb{A}^{f}\right)$. For any $\tau$ in $\operatorname{Aut}(\mathbb{C}),{ }^{\tau} \stackrel{d f}{=} \pi^{-1}(\tau \mid \Phi)$ is a right $\mathcal{G}$-torsor with a distinguished $\mathbb{A}^{f}$-point $\operatorname{sp}(\tau)$.

Let $\mathbf{C M}_{\mathbb{Q}}$ be the category of motives over $\Phi$ generated by abelian varieties of $C M$-type over $\Phi$ and the Tate motive, and let $\mathbf{C M}_{\mathbb{Q}}$ be the category of motives over $\mathbb{Q}$ generated by the abelian varieties over $\mathbb{Q}$ of potential $C M$-type, the Tate motive, and the Artin motives. The objects of these categories will be called $C M$-motives over $\Phi$ and $\mathbb{Q}$ respectively. Both categories are Tannakian and have natural $\mathbb{Q}$-linear fibre functors sending a motive $M$ to the Betti cohomology group of $\imath M$, and it is known that $\mathfrak{S}$ and $\mathfrak{I}$ are the pro-algebraic groups associated with $\mathbf{C M}_{\mathbb{Q}}$ and $\mathbf{C M}_{\mathbb{Q}}$ (see Deligne (1982b) for $\mathfrak{T}$ ). In particular, this means that with each $\mathbb{Q}$-linear representation $(r, V)$ of $\mathfrak{T}$ there is associated a $C M$-motive $M$ over $\mathbb{Q}$, well-defined up to a unique isomorphism. The Betti cohomology group $H_{B}(l M)=V$, and the Hodge structure on $H_{B}(l M)$ is defined by $r \circ h_{\text {can }}$. 
Let $M$ be a $C M$ motive over $\bar{Q}$. A tensor $t$ of $H_{B}(\imath M)$ will be called a Hodge cycle if there is a Hodge cycle $\left(t_{d R}, t_{e t}\right)$ of $H_{d R}(M) \times H_{e t}(M)$ on $M$ relative $l$ (in the sense of Deligne (1982a), p. 28) such that $t$ and $\left(t_{d R}, t_{e t}\right)$ have the same image as a tensor of $H_{d R}(l M) \times H_{e t}(l M)$. For any $\tau \in \mathrm{Gal}(\mathbb{Q} / \mathbb{Q})$, Deligne's theorem [ibid., 2.11] implies that there exists a Hodge cycle ${ }^{\gamma} t$ of $H_{B}(\tau M)$ corresponding to the tensor $\left(\tau t_{d R}, \tau t_{e t}\right)$ of $H_{d R}(\tau M) \times H_{e t}(\tau M)$. For example, when $t$ is the class of an algebraic cycle $Z$ on an abelian variety $A,{ }^{t} t$ is the class of $\tau Z$ on $\tau A$.

The functor $H_{d R}$ is a second fibre functor on $\mathbf{C M}_{\mathbb{Q}}$ with values in the category of vector spaces over $\mathbb{Q}$. Therefore $\mathscr{P}=\mathscr{H}_{\mathrm{cm}}{ }^{\otimes}\left(H_{B}, H_{d R}\right)$ is a torsor for $\mathfrak{T}$ (see Deligne and Milne (1982), 3.2), which we call the period torsor. The comparison isomorphisms $c(M): H_{B}(M) \otimes \mathbb{C} \stackrel{\approx}{\longrightarrow} H_{d R}\left(M_{\mathbb{C}}\right)$ define a canonical element $c$ in $\mathscr{P}(\mathbb{C})$. The $\mathbb{Q}$-structures $H_{B}(M)$ and $H_{d R}(M)$ determine actions of $\operatorname{Aut}(\mathbb{C})$ on $H_{B}(M) \otimes \mathbb{C}$ and $H_{d R}\left(M_{\mathbb{C}}\right)$, and for $\tau \in \operatorname{Aut}(\mathbb{C})$, we write

$$
z_{\infty}(\tau)=c^{-1} \circ \tau(c) \in \mathfrak{T}(\mathbb{C}) .
$$

The $\operatorname{map} z_{\infty}: \operatorname{Aut}(\mathbb{C}) \rightarrow \mathfrak{I}(\mathbb{C})$ is a one cocycle:

$$
z_{\infty}(\sigma \tau)=z_{\infty}(\sigma) \cdot \sigma z_{\infty}(\tau), \sigma, \tau \in \operatorname{Aut}(\mathbb{C}) .
$$

Note that $z_{\infty}$ does not factor through the quotient $\operatorname{Gal}(\Phi / \mathbb{Q})$ of $\operatorname{Aut}(\mathbb{C})$.

Proposition 1.1. The element $z_{\infty}(\tau)$ lies in $\tau \mathbb{S}(\mathbb{C})$.

Proof. We have to show that $z_{\infty}(\tau)$ maps to $\tau$ in $\operatorname{Gal}(\bar{Q} / \mathbb{Q})$. Recall that $\mathrm{Gal}(\bar{Q} / \mathbb{Q})$ is the group associated with the Tannakian category Art $_{\mathbb{Q}}$ of Artin motives over $\mathbb{Q}$, and that the map $\pi: \mathfrak{I} \rightarrow \operatorname{Gal}(\bar{Q} / \mathbb{Q})$ corresponds to the inclusion of Art $_{\mathbb{Q}}$ into $\mathbf{C M}_{\mathbb{Q}}$. It therefore suffices to show that, for all Artin motives $M$, $z_{\infty}(\tau)(M): H_{B}\left(M_{\mathbb{C}}\right) \otimes \mathbb{C} \rightarrow H_{B}\left(M_{\mathbb{C}}\right) \otimes \mathbb{C}$ is simply $\tau \otimes 1$.

Every Artin motive $M$ is a direct factor of a motive of the form $h(X)$ with $X$ a finite scheme over $\mathbb{Q}$ (see Deligne and Milne (1982), p. 211), and so we may suppose that $M=h(X)$. Then $H_{B}(M)=\operatorname{Hom}(X(\mathbb{Q}), \mathbb{Q})$ and $M$ corresponds to the representation of $\mathrm{Gal}(\Phi / \mathbb{Q})$ on $H_{B}(M)$ induced by its action on $X(\bar{Q})$. For any $\mathbb{Q}$-algebra $R, H_{d R}\left(M_{R}\right)=A \otimes R$, where $A=\Gamma\left(X, \mathcal{O}_{X}\right)$, and $A \otimes \Phi=\operatorname{Hom}(X(\Phi), \Phi)$ with $\operatorname{Gal}(\Phi / \mathbb{Q})$ acting on the second term through its action on $X(\Phi)$ and $\Phi$. In summary:

(a) $H_{B}(M)=\operatorname{Hom}(X(\Phi), \mathbb{Q})$ with $\operatorname{Gal}(\Phi / \mathbb{Q})$ acting through its action on $X(\Phi)$; $\mathbb{C}$;

(b) $H_{B}(M) \otimes \mathbb{C}=\operatorname{Hom}(X(\mathbb{Q}), \mathbb{C})$ with $\operatorname{Aut}(\mathbb{C})$ acting through its action on

(c) $H_{d R}\left(M_{\mathbb{C}}\right)=\operatorname{Hom}(X(\bar{Q}), \mathbb{C})$ with $\operatorname{Aut}(\mathbb{C})$ acting through its action on $X(\bar{Q})$ and $\mathbb{C}$. All actions are on the left. With these identifications, $c$ becomes the identity map, and for $\lambda \in \operatorname{Hom}(X(\Phi), \mathbb{C})=H_{B}(M) \otimes \mathbb{C}$,

$$
\begin{aligned}
z_{\infty}(\tau)(\lambda) & \stackrel{d f}{=}\left(c^{-1} \circ \tau c\right)(\lambda) \stackrel{d f}{=}\left(c^{-1} \circ \tau \circ c \circ \tau^{-1}\right)(\lambda) \\
& \stackrel{(b)}{=}\left(c^{-1} \circ \tau\right)\left(\tau^{-1} \circ \lambda\right) \stackrel{(c)}{=} \tau \circ\left(\tau^{-1} \circ \lambda\right) \circ \tau^{-1}=\lambda \circ \tau^{-1} \stackrel{(a)}{=}(\tau \otimes 1)(\lambda),
\end{aligned}
$$

as required. 
Remark 1.2. (a) We leave open the question of giving a description of $\mathscr{P}$ and its canonical $\mathbb{C}$-valued point (equivalently of $z_{\infty}(\tau)$ ) in the spirit of Langlands's definition of the Taniyama group (in particular, a description that avoids mentioning $C M$ motives). As Deligne pointed out to me, $\pi_{*}(\mathscr{P})$ is the $\operatorname{Gal}(\Phi / \mathbb{Q})$ torsor $\operatorname{Spec}(\bar{Q})$.

(b) It is possible to give a slightly different interpretation of $z_{\infty}(\tau)$. For a $C M$-motive $M$ over $\Phi$, write $H_{\tau}(M)=H_{B}(\tau \imath M)$. Then $H_{\tau}(M)$ is a fibre functor on $\mathbf{C M}_{\mathbb{Q}}$, and for any $\mathbb{Q}$-algebra $R,{ }^{\tau} \mathcal{S}(R)=H_{b} m{ }^{\otimes}\left(H_{i d} \otimes R, H_{\tau} \otimes R\right)$ (see Deligne and Milne (1982), 6.23). An element of ${ }^{\tau} \mathfrak{S}(\mathbb{C})$ is therefore a $\mathbb{C}$-linear functorial isomorphism $H_{B}(\imath M) \otimes \mathbb{C} \stackrel{\approx}{\longrightarrow} H_{B}(\tau \imath M) \otimes \mathbb{C}$, compatible with tensor products, and such that $t \otimes 1$ corresponds to ${ }^{i} t \otimes 1$ for all Hodge cycles $t$ of $H_{B}(i M)$. Consider the maps

$$
\begin{aligned}
& H_{B}(l M) \otimes \mathbb{C} \stackrel{1 \otimes \mathfrak{t}^{-1}}{\longrightarrow} H_{B}(l M) \otimes \mathbb{C} \stackrel{c(l M)}{\longrightarrow} H_{d R}(M) \otimes_{\mathbb{Q}, l} \mathbb{C} \\
& \stackrel{1 \otimes \tau}{\longrightarrow} H_{d R}(M) \otimes_{\mathbb{Q}, \tau \imath} \mathbb{C} \stackrel{c(\tau I M)^{-1}}{\longrightarrow} H_{B}(\tau I M) \otimes \mathbb{C} .
\end{aligned}
$$

Obviously the composite is $\mathbb{C}$-linear, and for any Hodge cycle $t$ of $H_{B}(l M)$,

$$
t \otimes 1 \leftrightarrow t \otimes 1 \leftrightarrow t_{d R} \otimes_{\mathbb{Q}, 1} 1 \leftrightarrow t_{d R} \otimes_{\mathbb{Q}, t t} 1 \leftrightarrow^{\tau} t \otimes 1 .
$$

Since the maps are functorial and compatible with tensor products, they define an element of ${ }^{\tau} \mathfrak{S}(\mathbb{C})$, which is clearly $z_{\infty}(\tau)$.

Let $(G, X)$ be a pair defining a connected Shimura variety, and let $x$ be a special point of $X$. By definition, this means that there is a (maximal) $\mathbb{Q}$-rational torus $T$ in $G$ such that $h_{x}$ factors through $(T / Z)(\mathbb{R}), Z=Z(G)$. From the universal property of $\Xi$, we know that there is a unique $\mathbb{Q}$-rational homomorphism $\rho_{x}: \Im \rightarrow T / Z$ such that $h_{x}=\left(\rho_{x}\right)_{\mathbb{R}} \circ h_{\text {can }}$. The map $\rho_{x}: \Im \rightarrow G^{\text {ad }}$ defines an action of $\mathfrak{G}$ on $G$, and the $\mathfrak{S}$-torsor ${ }^{\tau} \mathfrak{S}$ can be used to twist $G$ (or any other covering

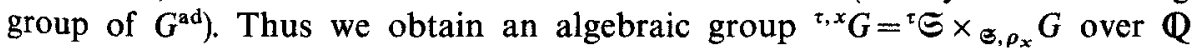
such that (as a $\mathrm{Gal}(\Phi / \mathbb{Q})$-set)

$$
{ }^{\tau, x} G(\bar{Q})=\left\{s \cdot g \mid s \in{ }^{\tau} \circlearrowleft(\bar{Q}), g \in G(\bar{Q})\right\} / \Im(\bar{Q})
$$

where $s_{1} \in S(\Phi)$ acts according to the rule

$$
(s \cdot g) s_{1}=s s_{1} \cdot \operatorname{ad}\left(\rho_{x}\left(s_{1}^{-1}\right)\right) g .
$$

Then ${ }^{\tau, x} G$ is a semisimple group having ${ }^{\tau} \mathfrak{S} \times \times_{\Im, \rho_{x}} T=T$ as a subtorus.

Let $q E^{\tau, x} G^{\text {ad }}(\mathbb{Q})$, and let $s \in \rho_{x *}\left({ }^{\tau} \Theta\right)(\Phi)$; then $q$ can be written $\left[s \cdot q_{s}\right]$ for a unique element $q_{s} \in G^{\text {ad }}(\overline{\mathbb{Q}})$, and $s \mapsto s q_{s}$ defines a $\mathbb{Q}$-rational automorphism (q) of $\rho_{x *}\left({ }^{\tau} \Xi\right)$ (as a $G^{\text {ad }}$-torsor). In this way, ${ }^{\tau, x} G$ can be identified with the group of automorphisms of the $G$-torsor $\rho_{x *}\left({ }^{\tau} \Xi\right)$ (acting on the right).

The point $\operatorname{sp}(\tau)$ in ${ }^{\tau} \subseteq\left(\mathbb{A}^{f}\right)$ defines a canonical continuous isomorphism

$$
g \mapsto^{\tau, x} g=[\operatorname{sp}(\tau) \cdot g]: \tilde{G}\left(\mathbb{A}^{f}\right) \rightarrow^{\tau, x} \tilde{G}\left(\mathbb{A}^{f}\right) .
$$


In (Milne and Shih (1982b), 8.2) it is shown how to construct a canonical isomorphism

$$
g \mapsto{ }^{\tau, x} g: G^{\text {ad }}(\mathbb{Q})^{+\wedge} \rightarrow^{\tau, x} G^{\text {ad }}(\mathbb{Q})^{+\wedge}
$$

compatible with the preceding isomorphism (cf. (3.7) below). Define ${ }^{\tau} h$ to be the homomorphism $\mathbf{S} \rightarrow^{\tau_{x} x} G_{\mathbb{R}}^{\text {ad }}$ associated with the cocharacter $\tau \mu_{x}$ of $T / Z \subset{ }^{\tau, x} G^{\text {ad }}$. If we let ${ }^{\tau, x} X$ denote the ${ }^{\tau, x} G^{\text {ad }}(\mathbb{R})^{+}$-conjugacy class of maps $\mathbf{S} \rightarrow{ }^{\tau, x} G_{\mathbf{R}}^{\text {ad }}$ containing ${ }^{\tau} h$, then the pair $\left({ }^{\tau, x} G,{ }^{\tau, x} X\right)$ again defines a connected Shimura variety. We write ${ }^{\tau} x$ for ${ }^{\tau} h$ regarded as a point of ${ }^{\tau, x} X$.

Proposition 1.3. For any special points $x$ and $x^{\prime}$ of $X$, there is a canonical isomorphism

$$
\varphi^{0}\left(\tau ; x^{\prime}, x\right): S^{0}\left({ }^{\tau, x} G,{ }^{\tau, x} X\right) \rightarrow S^{0}\left({ }^{\tau, x^{\prime}} G,{ }^{\tau, x^{\prime}} X\right)
$$

such that $\varphi^{0}\left(\tau ; x^{\prime}, x\right) \circ\left(^{\tau, x} g\right)=\left({ }^{\tau, x}{ }^{\prime} g\right) \circ \varphi^{0}\left(\tau ; x^{\prime}, x\right)$ for all $g \in G^{\text {ad }}(\mathbb{Q})^{+\wedge}$.

Proof. Because $\widetilde{G}^{\text {ad }}(\mathbb{Q})^{+\wedge} \rightarrow G^{\text {ad }}(\mathbb{Q})^{+\wedge}$ is surjective, and $S^{0}(G, X)$ is the quotient of $S^{\circ}(\widetilde{G}, X)$ by the kernel of this map, it suffices to prove the proposition for $(\tilde{G}, X)$. Thus we may assume that $G$ is simply connected.

Lemma 1.4. Let $(G, X)$ and $\left(G^{\prime}, X^{\prime}\right)$ be pairs defining connected Shimura varieties. Let $f$ and $f^{\prime}$ be isomorphisms

$$
f: G \stackrel{\approx}{\longrightarrow} G^{\prime}, \quad f^{\prime}: G^{\text {ad }}(\mathbb{Q})^{+\wedge} \stackrel{\approx}{\longrightarrow} G^{\text {ad }}(\mathbb{Q})^{+\wedge},
$$

and let $\gamma \in G^{\mathrm{ad}}(\mathbb{Q})^{+\wedge}$ be such that, when $f$ is extended by continuity to an isomorphism $\hat{f}: G^{\text {ad }}(\mathbb{Q})^{+\wedge} \stackrel{\approx}{\longrightarrow} G^{\text {add }}(\mathbb{Q})^{+\wedge}$, then $\hat{f} \circ \operatorname{ad}(\gamma)=f^{\prime}$. Under these conditions

$$
\varphi \stackrel{d f}{=} S^{0}\left(f_{1}\right) \circ(\gamma): S^{0}(G, X) \rightarrow S^{0}\left(G^{\prime}, X^{\prime}\right)
$$

is an isomorphism such that $\varphi \circ(a)=\left(f^{\prime}(a)\right) \circ \varphi$ for all $a \in G^{\text {ad }}(\mathbb{Q})^{+\wedge}$; moreover, if $f$ is replaced with $f \circ \operatorname{ad}(q), q \in G^{\mathrm{ad}}(\mathbb{Q})^{+}$, and $\gamma$ with $q^{-1} \gamma$, then $\varphi$ is unchanged.

Proof. Straightforward.

Thus we must find a pair $(f, \gamma)$, well-defined up to replacement by $\left(f \circ \operatorname{ad}(q), q^{-1} \gamma\right)$ with $q \in^{\tau, x} G^{\text {ad }}(\mathbb{Q})^{+}$, such that

(i) $f$ is an isomorphism ${ }^{\tau, x} G \rightarrow{ }^{\tau, x^{\prime}} G$ sending ${ }^{\tau, x} X$ into ${ }^{\tau, x^{\prime}} X$;

(ii) $\gamma \in^{\tau, x} G^{\text {ad }}(\mathbb{Q})^{+\wedge}$ satisfies $f\left(\operatorname{ad}(\gamma)\left({ }^{\tau, x} a\right)\right)={ }^{\tau, x^{\prime}} a$ for all $a \in G^{\text {ad }}(\mathbb{Q})^{+\wedge}$.

Let $c$ be the class of ${ }^{\tau} \mathfrak{S}$ in $H^{1}(\mathbb{Q}, \subseteq)$. The existence of the section sp shows that $c$ maps to zero in $H^{1}\left(\mathbb{Q}_{l}, \subseteq\right)$ for all primes $l$. Consider $\rho_{x}(c)$ and $\rho_{x^{\prime}}(c) \in H^{1}\left(\mathbb{Q} G^{\text {ad }}\right)$. These elements have the same image in $H^{1}\left(\mathbb{R}, G^{\text {ad }}\right)$; this can be proved by a direct calculation, which is carried out in (Milne and Shih (1982b), pp. 315-316), or by noting that (Deligne (1979), 1.2.2) implies that ${ }^{\tau, x} G_{\mathbf{R}}^{\text {ad }} \approx^{\tau, x^{\prime}} G_{\mathbf{R}}^{\text {ad }}$. Since the Hasse principle holds for $G^{\text {ad }}$ (see for example (Milne (1986), I.9.9), it follows that $\rho_{x}(c)$ and $\rho_{x^{\prime}}(c)$ are equal. 
Choose a pair $\left(G_{1}, X_{1}\right)$ as in (A.4) of the appendix. The constructions reviewed above for $(G, X)$ have analogues for $\left(G_{1}, X_{1}\right)$. We write $x_{1}$ and $x_{1}^{\prime}$ for $x$ and $x^{\prime}$ regarded as points of $X_{1}$ when it is necessary to make this distinction. They are special, and (A.4b) implies they give rise to homomorphisms $\rho_{x_{1}}, \rho_{x^{\prime}}: \subseteq \rightarrow G_{1}$ lifting $\rho_{x}$ and $\rho_{x^{\prime}}$. Because of (A.4c), the map $H^{1}\left(\mathbb{Q}, G_{1}\right) \rightarrow H^{1}\left(\mathbb{Q}, G_{1}^{\text {ad }}\right)$ is injective, and so $\rho_{x_{1 *}}\left({ }^{\tau} \mathcal{S}\right)$ and $\rho_{x_{1}^{\prime}}\left({ }^{\tau} \Xi\right)$ are isomorphic $G_{1}$-torsors. The choice of an isomorphism $t: \rho_{x_{1} *}\left({ }^{\tau} \Xi\right) \stackrel{\approx}{\longrightarrow} \rho_{x_{1}^{\prime} *}\left({ }^{(} \Xi\right)$ determines an isomorphism $f_{1}:{ }^{\tau, x} G_{1} \stackrel{\approx}{\longrightarrow}{ }^{\tau, x^{\prime}} G_{1}$, and it is known (Langlands (1979), p. 232) that $f_{1}$ maps ${ }^{\tau, x} X_{1}$ into ${ }^{\tau, x^{\prime}} X_{1}$. From the discussion preceding the statement of the proposition, we know that $t$ is determined up to replacement by an element $t \circ(q), q{ }^{\tau, x} G_{1}(\mathbb{Q})$. Since ${ }^{\tau, x} G_{1}(\mathbb{Q})$ is dense in ${ }^{\tau, x} G_{1}(\mathbb{R})$, we can modify $t$ so that $f_{1}$ maps the component ${ }^{\tau, x} X$ of ${ }^{t, x} X_{1}$ into the component ${ }^{\tau, x^{\prime}} X$ of ${ }^{\tau, x^{\prime}} X_{1}$. Then $f_{1}$ restricts to a morphism $f:\left({ }^{\tau, x} G,{ }^{\tau, x} X\right) \rightarrow\left({ }^{\tau, x}{ }^{\prime} G,{ }^{\tau, x^{\prime}} X\right)$, and when $t$ is replaced by $t \circ(q)$, $q \in{ }^{\tau, x} G_{1}(\mathbb{Q})_{+}$, then $f$ is replaced by $f \circ \operatorname{ad}(\bar{q})$ where $\bar{q}$ is the image of $q$ in ${ }^{\tau, x} G^{\text {ad }}(\mathbb{Q})^{+}$.

If $s$ and $s^{\prime}$ denote the images of $\operatorname{sp}(\tau)$ in $\rho_{x_{1} *}\left({ }^{\tau} \mathbb{S}\right)\left(\mathbb{A}^{f}\right)$ and $\rho_{x_{1} *}\left({ }^{\tau} \subseteq\right)\left(\mathbb{A}^{f}\right)$ respectively, then there exists an element $\gamma_{1} \in^{\tau, x} G_{1}\left(\mathbb{A}^{f}\right)$ such that $t\left(s \gamma_{1}\right)=s^{\prime}$. Note that $\gamma_{1}$ is uniquely determined by the choice of $t$, and that if $t$ is replaced by $t \circ(q), q \in^{\tau, x} G_{1}(\mathbb{Q})_{+}$, then $\gamma_{1}$ is replaced by $q^{-1} \gamma_{1}$. Note also that $f_{1} \circ \operatorname{ad}\left(\gamma_{1}\right)\left(^{\tau, x} a\right)$ $={ }^{\imath, x^{\prime}} a$ for all $a \in G_{1}\left(\mathbb{A}^{f}\right)$. We shall show that the image $\gamma$ of $\gamma_{1}$ in ${ }^{\tau, x} G_{1}\left(\mathbb{A}^{f}\right) / Z_{1}(\mathbb{Q})^{-}$lies in the subgroup ${ }^{\tau, x} G^{\text {ad }}(\mathbb{Q})^{+\wedge}($ cf. $\S 0)$. Since the maps $a \mapsto^{\tau, x} a$ and $a \mapsto^{\tau, x^{\prime}} a$ on $G_{1}\left(\mathbb{A}^{f}\right)$ induce the maps of the same name on the subquotient $G^{\text {ad }}(\mathbb{Q})^{+\wedge}$ of $G_{1}\left(\mathbb{A}^{f}\right)$ (Milne and Shih $\left.(1982 \mathrm{~b}), 8.2\right)$, the lemma will then show that the pair $(f, \gamma)$ defines a map $\varphi^{0}\left(\tau ; x^{\prime}, x\right)$ having the desired properties. It is independent of the choice of $t$, and the usual argument [ibid. p. 339-340] shows that it is independent of the choice of $\left(G_{1}, X_{1}\right)$.

Write $G_{1}^{\prime}$ for ${ }^{\tau, x} G_{1}$. We shall show that $\gamma_{1}$ lies in the closure of $G_{1}^{\prime}(\mathbb{Q})^{+-}$ of $G_{1}^{\prime}(\mathbb{Q})^{+}$in $G_{1}^{\prime}\left(\mathbb{A}^{f}\right)$. Recall (Deligne (1979), 2.5.1) that $G_{1}^{\prime}(\mathbb{Q})^{+-}$is the fibre over 1 of $G_{1}^{\prime}\left(\mathbb{A}^{f}\right) \rightarrow \pi_{0}\left(G_{1}^{\prime}(\mathbb{Q}) \backslash G_{1}^{\prime}(\mathbb{A})\right.$ ), and (Deligne $(1971)$, 2.4) that $\pi_{0}\left(G_{1}^{\prime}(\mathbb{Q}) \backslash G_{1}^{\prime}(\mathbb{A})\right) \stackrel{\approx}{\longrightarrow} \pi_{0}\left(H_{1}(\mathbb{Q}) \backslash H_{1}(\mathbb{A})\right)$ where $H_{1}=G_{1}^{\prime} / G_{1}^{\prime \text { der }}=G_{1} / G_{1}^{\text {der }}$. The maps $\rho_{x_{1}}$ and $\rho_{x_{1}^{\prime}}$ become equal when composed with $v: G_{1} \rightarrow H_{1}$, and so, when the above constructions are carried out with $G_{1}$ replaced with $H_{1}$, one sees immediately that the image of $\gamma_{1}$ in $H_{1}\left(\mathbb{A}^{f}\right)$ lies in $H_{1}(\mathbb{Q})$. Therefore $\gamma$ lies in $G_{1}(\mathbb{Q})^{+-}$.

Remark 1.5. In $\S 3$ we shall need to use a slight strengthening of some of the above arguments. Let $H_{1}(\mathbb{R})_{+}$be the image of $Z_{1}(\mathbb{R})$ in $H_{1}(\mathbb{R})$ and let $H_{1}(\mathbb{Q})_{+}=H_{1}(\mathbb{Q}) \cap H_{1}(\mathbb{R})_{+}$. Write $\rho$ for $\nu \circ \rho_{x_{1}}=\nu \circ \rho_{x_{1}^{\prime}}$. An isomorphism $t: \rho_{x_{1 *}}\left({ }^{\tau} \Xi\right)-\stackrel{\approx}{\longrightarrow} \rho_{x_{1}^{\prime} *}\left({ }^{\tau} \Xi\right)$ induces an automorphism of $\rho_{*}\left({ }^{\top} \Xi\right)$, and hence defines an element $q \in H_{1}(\mathbb{Q})$. If $t$ is replaced by $t \circ(g), g \in^{\tau, x} G_{1}(\mathbb{Q})_{+}$, then $q$ is replaced by $q \cdot v(g)$. Since $v\left(G_{1}(\mathbb{R})_{+}\right) \subset v\left(Z_{1}(\mathbb{R})\right)$, we see that we obtain an element $q_{1} \in H_{1}(\mathbb{Q}) / H_{1}(\mathbb{Q})_{+}$which is independent of the choice of $t$. Probably it is possible to show in general that this is 1 by refining the proof of the "Second Lemma 
of Comparison" in Langlands (1979), p. 232, but we shall use a different argument to prove this in the cases of immediate interest to us.

The construction in (A.2) leads to a pair $\left(G_{1}, X_{1}\right)$ such that the weight is in fact zero. Let $G_{0}$ be the subgroup of $G_{1}$ constructed in (A.5), and let $H_{0}=G_{0} / G$. Write $x_{0}$ and $x_{0}^{\prime}$ for $x$ and $x^{\prime}$ regarded as homomorphisms $\mathbf{S} \rightarrow G_{0, \mathbb{R}}$. Then $\rho_{x_{0} *}\left({ }^{\tau} \subseteq\right)$ and $\rho_{x^{6} *}\left({ }^{\tau} \subseteq\right)$ are isomorphic $G_{0}$-torsors (see the argument in (Milne and Shih (1982b), pp. 315-316)). This shows that there is an element $q_{0} \in H_{0}(\mathbb{Q}) / H_{0}(\mathbb{Q})_{+}$mapping to $q_{1} \in H_{1}(\mathbb{Q}) / H_{1}(\mathbb{Q})_{+}$. But $H_{0 \mathbb{R}} / w\left(\mathbb{G}_{m}\right)$ is anisotropic, and is therefore connected. Since $w\left(\mathbb{G}_{m}\right)=1, H_{0}(\mathbb{R})$ is itself connected, and so $v\left(Z_{0}(\mathbb{R})\right)=H_{0}(\mathbb{R})$. Therefore that $q_{0}=1$, as required.

Theorem 1.6. For each $\tau \in \operatorname{Aut}(\mathbb{C})$, there is a unique isomorphism

$$
\varphi_{\tau, x}^{0}: \tau S^{0}(G, X) \rightarrow S^{0}\left({ }^{\tau, x} G,{ }^{\mathfrak{t}, x} X\right)
$$

such that

(i) the point $\tau[x]$ is mapped to $\left[{ }^{\tau} x\right]$;

(ii) $\varphi_{\tau, x^{\circ}}^{0} \tau(g)=(\tau, x) \circ \varphi_{\tau, x}^{0}$ for all $g \in G^{\text {ad }}(\mathbb{Q})^{+\wedge}$. Moreover, if $X^{\prime}$ is a second special point of $X$, then

$$
\varphi^{0}\left(\tau ; x^{\prime}, x\right) \circ \varphi_{\tau, x}^{0}=\varphi_{\tau, x^{\prime}}^{0} .
$$

Proof. See Milne and Shih (1982b) for Shimura varieties of abelian type and Milne (1983) for the general case.

Remark 1.7. Theorem 1.6 has a down-to-earth interpretation. Let $(G, X)$ be a pair defining a connected Shimura variety, and let $f$ be an automorphic function on $X$ relative to a congruence subgroup $\Gamma$ of $G(\mathbb{Q})$. We can regard $f$ as an algebraic function on $\Gamma \backslash X$. By definition, $\Gamma=K \cap G(\mathbb{Q})$ for some compact open subgroup $K$ in $G\left(\mathbb{A}^{f}\right)$, and we define the congruence subgroup ${ }^{\tau, x} \Gamma$ of ${ }^{\tau, x} G(\mathbb{Q})$ to be ${ }^{\tau, x} K \cap^{\tau, x} G(\mathbb{Q})$ where ${ }^{\tau, x} K$ is the image of $K$ under the isomorphism $g \mapsto^{\tau, x} g: G\left(\mathbb{A}^{f}\right) \stackrel{\approx}{\longrightarrow}, x \in\left(\mathbb{A}^{f}\right)$. Let $\tau$ be an automorphism of $\mathbb{C}$, and let $x$ be a special point of $X$. Then the theorem defines an isomorphism

$$
\left(\varphi_{\tau, x}^{0}\right)_{\Gamma}: \tau(\Gamma \backslash X) \rightarrow^{\tau, x} \Gamma \backslash{ }^{\tau, x} X .
$$

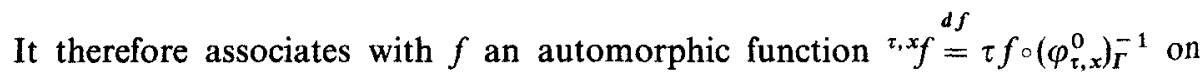
the Hermitian symmetric domain ${ }^{\tau, x} X$ relative to ${ }^{\tau, x} \Gamma$ such that

$$
{ }^{\tau, x} f\left(\left[{ }^{\tau} x\right]\right)=\tau(f([x]))(\text { for the chosen special point } x) .
$$

Moreover, ${ }^{\tau, x}(f \circ(g))={ }^{\tau, x} f \circ\left({ }^{\tau, x} g\right)$ for all $g \in G^{\text {ad }}(\mathbb{Q})^{+\wedge}$.

Let $G$ be a reductive algebraic group over $\mathbb{Q}$, and let $\rho$ be a homomorphism $\mathfrak{S} \rightarrow G$. It is possible to give a motivic description of ${ }^{\tau, \rho} G \stackrel{d f}{=}{ }^{\tau} \mathfrak{S} \times{ }_{\mathfrak{S}, \rho} G$. Choose a faithful $\mathbb{Q}$-linear representation $r: G \hookrightarrow G L(V)$ of $G$. There exists a family of tensors $\left(t_{\alpha}\right)_{\alpha \in A}$ of $V$ (that is, elements of vector spaces of the form $T_{j}^{i V}$ $\left.=V^{\otimes i} \otimes(\bar{V})^{\otimes j}\right)$ such that $G$ is the subgroup of $G L(V)$ fixing the $t_{\alpha}$. If $M$ is the $C M$-motive over $\Phi$ corresponding to the representation $r \circ \rho$, then $H_{B}(M)=V$ 
and the $t_{\alpha}$ are Hodge cycles on $M$. Write ${ }^{\tau, \rho} V={ }^{\tau} \mathbb{S} \times{ }_{\mathfrak{S}, \rho} V$, so that (as $\mathrm{Gal}(\mathbb{Q} / \mathbb{Q})$ )modules)

$$
{ }^{\tau, \rho} V \otimes \Phi=\left\{s \cdot v \mid s \in^{\tau} \Xi(\bar{\Phi}), g \in V \otimes \Phi\right) / \Im(\Phi)
$$

where $s_{1} \in \mathbb{S}(\Phi)$ acts according to the rule

$$
(s \cdot v) s_{1}=s s_{1} \cdot \rho\left(s_{1}^{-1}\right) v .
$$

Proposition 1.8. There is a canonical isomorphism ${ }^{\tau, \rho} V \approx H_{B}(\tau M)$, and ${ }^{\tau, \rho} G$ is the subgroup of $G L\left(H_{B}(\tau M)\right)$ fixing the Hodge cycles ${ }^{\tau} t_{\alpha}$ on $\tau M$.

Proof. Let $\Xi$ be the image of $\subseteq$ in $G L\left(H_{B}(M)\right)$, and let ${ }^{\tau} \bar{\Xi}$ be the $\Xi$-torsor defined by ${ }^{\top} \mathcal{G}$. For any $\mathbb{Q}$-algebra $R$, the $R$-valued points of ${ }^{\top} \mathbb{G}$ can be identified with the set of $R$-linear maps $H_{B}(M) \otimes R \rightarrow H_{B}(\tau M) \otimes R$ taking $t$ to ${ }^{\tau} t$ for all Hodge cycles $t$ on $M$ (see Deligne and Milne (1982), especially (6.23a)). Clearly ${ }^{\tau, \rho} V={ }^{\tau} \widetilde{G} \times_{\bar{\xi}, \rho} V$, and the map $[s \cdot v] \rightarrow s(v)$ identifies this with $H_{B}(\tau M)$. Choose a point $s_{0} \in \tilde{\tau}(\Phi)$, and consider the map

$$
\operatorname{Aut}\left(H_{B}(\tau M) \otimes \Phi,\left({ }^{\tau} t_{\alpha}\right)_{\alpha \in A}\right) \rightarrow^{\tau, \rho} G(\Phi), \quad a \mapsto\left[s_{0} \cdot \operatorname{ad}\left(s_{0}^{-1}\right) a\right] .
$$

One checks immediately that this is independent of the choice of $s_{0}$, that it defines an isomorphism, and that it commutes with the action of $\operatorname{Gal}(\overline{\mathbb{Q}} / \mathbb{Q})$. It therefore defines an isomorphism of algebraic groups over $\mathbb{Q}$.

\section{The compact dual symmetric Hermitian space and its conjugates}

We first review Deligne's interpretation (Deligne 1979) of a Hermitian symmetric domain $X$ and its compact dual $\check{X}$ as parameter spaces for Hodge structures and filtrations. Then we prove analogues of (1.3) and (1.6) for $\check{X}$.

Let $V$ be a vector space over a field $k$ of characteristic zero. A homomorphism $\mu: \mathbb{G}_{m} \rightarrow G L(V)$ defines a decomposition

$$
V=\oplus V^{i}, \quad V^{i}=\left\{v \in V \mid \mu(z) v=z^{i} v, \text { all } z \text { in } k^{\times}\right\}
$$

and a decreasing filtration of $V$

$$
\ldots F^{p} V \supset F^{p+1} V \ldots, \quad F^{p} V=\underset{i \geqq p}{\bigoplus} V^{i} .
$$

Let $G$ be a reductive group over $k$. A homomorphism $\mu: \mathbb{G}_{m} \rightarrow G$ defines a filtration $F^{*}$ on $V$ for each representation $(r, V)$ of $G$, namely, that corresponding to $r \circ \mu$. These filtrations are compatible with the formation of tensor products and duals. Conversely, any functor $(r, V) \mapsto\left(F^{*}, V\right)$ from representations of $G$ to filtrations compatible with tensor products and duals arises from a homomorphism $\mu: \mathbb{G}_{m} \rightarrow G$. We call such a functor a filtration of $\operatorname{Rep}_{k}(G)$, and we write Filt $(\mu)$ for the filtration defined by $\mu$.

Proposition 2.1. Let $G$ be a reductive group over a field $k$ of characteristic zero, and let $\mu$ be a cocharacter of $G$. From the adjoint action of $G$ on $\mathfrak{g}=\operatorname{Lie}(G)$, we acquire a filtration $F^{*}$ of $\mathfrak{g}$. 
(a) The subalgebra $F^{0} \mathrm{~g}$ is the Lie algebra of a parabolic subgroup $F^{0} G$ of $G$; moreover, $F^{0} G$ is the subgroup of $G$ respecting the filtration (defined by $\mu$ ) on each representation of $G$.

(b) The subalgebra $F^{1} \mathrm{~g}$ is the Lie algebra of the unipotent radical $F^{1} G$ of $F^{0} G$; moreover $F^{1} G$ is the subgroup of $F^{0} G$ that acts trivially on the graded module $\otimes\left(F^{p} V / F^{p+1} V\right)$ associated with each representation $(r, V)$ of $G$.

(c) The centralizer $Z(\mu)$ of $\mu$ is a Levi subgroup of $F^{0} G$; in particular, $Z(\mu) \stackrel{\approx}{\longrightarrow} F^{0} G / F^{1} G$, and the composite $\bar{\mu}$ of $\mu$ with $F^{0} G \rightarrow F^{0} G / F^{1} G$ is central. Two cocharacters of $G$ define the same filtration of $G$ if and only if they define the same group $F^{0} G$ and induce the same map $\mathbb{G}_{m} \rightarrow F^{0} G / F^{1} G$.

Proof. See (Saavedra (1972), especially IV.2.2.5).

Let $G$ be a reductive group over $\mathbb{C}$, and let $\mu_{0}: \mathbb{G}_{m} \rightarrow G$ be a cocharacter of $G$. We let $\breve{X}$ be the set of filtrations of $\operatorname{Rep}_{\mathbb{C}}(G)$ that are $G(\mathbb{C})$-conjugate to Filt $\left(\mu_{0}\right)$.

Proposition 2.2. The action

$$
G(\mathbb{C}) \times \check{X} \rightarrow \check{X}, \quad(g, \mu) \mapsto \text { Filt }(\operatorname{ad}(g) \circ \mu)
$$

defines a bijection $G(\mathbb{C}) / P_{0}(\mathbb{C}) \stackrel{\approx}{\longrightarrow}$, where $P_{0}$ is the parabolic subgroup $F^{0} G$ of $G$.

Proof. We have to show that $P_{0}(\mathbb{C})$ is the subgroup fixing Filt $\left(\mu_{0}\right)$ under the above action, but the filtration on a vector space $V$ defined by $\operatorname{ad}(g) \circ \mu_{0}$ is obtained from the filtration defined by $\mu_{0}$ by applying $g$, and so this follows from (2.1 a).

Remark 2.3. (a) The bijection in (2.2) endows $\check{X}$ with a complex structure. In fact, because $P_{0}$ is parabolic, $\check{X}$ has the structure of a smooth projective variety over $\mathbb{C}$.

(b) According to $(2.1 \mathrm{c})$, the points of $\breve{X}$ can be identified with the set of equivalence classes $[P, \mu]$, where $P$ is a parabolic subgroup of $G$ and $\mu$ is a cocharacter $\mu$ of $P$ such that $(P, \mu)$ is conjugate under $G(\mathbb{C})$ to $\left(P_{0}, \mu_{0}\right)$; the classes $[P, \mu]$ and $\left[P^{\prime}, \mu^{\prime}\right]$ are equal if and only if $P=P^{\prime}$ and $\mu$ and $\mu^{\prime}$ define the same cocharacter of $P / R_{u} P$.

(c) For any faithful representation $(r, V)$ of $G$ it is obvious that $\check{X}$ can be identified with the set of filtrations of $V$ conjugate to that defined by $\mu_{0}$. Slightly less obviously, $\breve{X}$ can also be identified with the set of filtrations of $\operatorname{Lie}(G)$ conjugate to that defined by $a d \circ \mu_{0}$.

By a global tensor of a sheaf $\mathscr{F}$ of $\mathcal{O}_{s}$-modules, we mean an element of $\Gamma\left(S, T_{j}^{i} \mathscr{V}\right)$ for some $i$ and $j$, where $T_{j}^{i} \mathscr{V}=\mathscr{V}^{\otimes i} \otimes \check{\mathscr{V}} \otimes j$.

Proposition 2.4. Let $\mu_{0}$ be a cocharacter of a reductive group $G$ over $\mathbb{C}$, and let $\check{X}=G(\mathbb{C}) / P_{0}(\mathbb{C})$ be the corresponding space of filtrations; let $r: G \rightarrow G L(V)$ be a faithful representation of $G$, and let $\mathscr{V}$ be the constant vector bundle $\check{X} \times V$ on $\bar{X}$. 
(a) There is a unique filtration of $\mathscr{V}$ by algebraic subbundles such that the filtration on each fibre $\mathscr{V}_{[P, \mu]}$ is that defined by $\mu$.

(b) Let $\left(t_{\alpha}\right)$ be a family of tensors of $V$ such that $G$ is the subgroup of $G L(V)$ fixing the $t_{\alpha}$. Let $F^{*}$ be a filtration (by algebraic subbundles) of the constant vector bundle $\mathscr{V}^{\prime}=S \times V$ on a smooth complex variety $S$, and let $t_{a}^{\prime}$ be the global tensor $\left(1, t_{\alpha}\right)$ of $\mathscr{V}^{\prime}$. If for each $s$ in $S$, there is an isomorphism $\mathscr{V}_{s}^{\prime} \approx V$ of filtered vector spaces mapping each $t_{\alpha, s}^{\prime}$ to $t_{\alpha}$, then there is a unique morphism $\gamma: S \rightarrow \check{X}$ such that when we identify $\gamma^{*} \mathscr{V}$ with $S \times V=\mathscr{V}^{\prime}$ in the obvious way, the filtrations on $\gamma^{*} \mathscr{V}$ and $\mathscr{V}^{\prime}$ agree.

Proof. (a) Let $\mathfrak{F}(V)$ be the flag variety of filtrations on $V$ conjugate under $G L(V)$ to that defined by $\mu_{0}$. Then the map sending Filt $(\mu)$ to the filtration on $V$ defined by $\mu$ is a closed immersion of $\check{X}$ into $\mathscr{F}(V)$, and the pullback of the universal bundle on $\mathfrak{F}(V)$ is $\mathscr{V}$. Clearly the filtration on the universal bundle induces the correct filtration on $\mathscr{r}$.

(b) For $y \in \mathfrak{F}(V)$, let $F_{y}^{*}$ be the corresponding filtration of $V$. Then $y$ is in the image of $\check{X}$ in $\widetilde{F}(V)$ if and only if there is an isomorphism of filtered vector spaces $\left(V, F_{0}\right) \stackrel{\approx}{\longrightarrow}\left(V, F_{y}\right)$ fixing each $t_{\alpha}$ because such isomorphisms are defined by elements of $G(\mathbb{C})$. Therefore the map $S \rightarrow \mathfrak{F}(V)$ defined by $\mathscr{V}$ and the universal property of $\mathfrak{F}(V)$ factors through $\breve{X}$, and the resulting map $S \rightarrow \check{X}$ has the correct properties.

Now let $(G, X)$ be a pair defining a connected Shimura variety.

Proposition 2.5. Let $r$ : $G_{\mathbb{R}}^{\text {ad }} \hookrightarrow G L(V)$ be a faithful real representation of $G^{\text {ad }}$, and let $\mathbf{V}$ be the constant sheaf on $X$ defined by $V$.

(b) For each $x$ in $X, r \circ h_{x}$ is a Hodge structure on the stalk $\mathbf{V}_{x}(=V)$ of $\mathbf{V} ; \mathbf{V}$, together with these Hodge structures, is a variation of real Hodge structures on $X$.

(b) There exists a bilinear form $\psi: V \times V \rightarrow \mathbb{R}$ defining a polarization of the real Hodge structure $\left(r \circ h_{x}, V\right)$ for all $x$.

(c) Let $\left\{t_{\alpha}\right\}$ be a family of tensors of $V$ such that $G_{\mathbb{R}}^{\text {ad }}$ is the subgroup of $G L(V)$ fixing the $t_{\alpha}$. Then as $x$ runs through the points of $X$, the Hodge structures $V_{x}$ run through a connected component of the set of all Hodge structures on $V$ for which the tensors $t_{\alpha}$ are all of type $(0,0)$.

Proof. See Deligne (1979), 1.1.14.

We now apply (2.2) and the preceding discussion to the group $G^{\text {ad }}$ and the cocharacter $\mu_{0}$ corresponding to a point $o \in X$. In particular, we define the $u^{\prime}$ iait space $\check{X}$ to be the set of filtrations of $\operatorname{Rep}_{\mathbb{C}}\left(G^{\text {ad }}\right)$ conjugate under $G^{\text {ad }}(\mathbb{C})$ to $\mu_{0}$ (and hence to all $\mu_{x}$ for $x$ in $X$ ). It is the compact dual symmetric Hermitian space of $X$ in the usual sense (Helgason 1978, V.2). Note that $G(\mathbb{C})$ acts on $\dot{X}$ through $G(\mathbb{C}) \rightarrow G^{\text {ad }}(\mathbb{C})$.

Proposition 2.6. The map $\beta: X \rightarrow \breve{X}$ sending a point $x$ in $X$ to the filtration of $\operatorname{Rep}_{\mathbb{C}}\left(G_{\mathbb{C}}^{\text {ad }}\right)$ defined by $\mu_{x}$ embeds $X$ as an open complex submanifold of $\breve{X}$. For $o \in X$, let $K_{0}$ be the isotropy group at $o$ in $G(\mathbb{R})^{+}$, and let $P_{0}$ be the isotropy group at $o \in \check{X}$ in $G(\mathbb{C})$; then $K_{0}=P_{0} \cap G(\mathbb{R})^{+}$, and the inclusion of $K_{0}$ into $P_{0}$ 
identifies $\left(K_{0}\right)_{\mathbb{C}}$ with a Levi subgroup of $P_{0}$; there is an equivariant commutative diagram

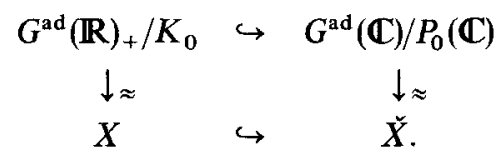

Proof. This is proved in Deligne (1979), 1.1.14. We merely note that the injectivity of $X \rightarrow \check{X}$ follows from the fact that the Hodge filtration determines the Hodge decomposition.

The map $\beta$ is the Borel embedding of $X$ into $\breve{X}$. Since $\breve{X}$ is an algebraic variety, we can speak of the variety $\tau \check{X}$ for $\tau$ an automorphism of $\mathbb{C}$. We shall show that $\tau \check{X}$ has a natural realization as the dual of a Hermitian symmetric domain.

Recall that for all automorphisms $\tau$ of $\mathbb{C}$, we have a canonical element $z_{\infty}(\tau) \in^{\tau} \mathfrak{G}(\mathbb{C})$. This gives rise to a canonical map

$$
g \mapsto^{t, x} g \stackrel{d f}{=}\left[z_{\infty}(\tau) \cdot g\right]: G^{\prime}(\mathbb{C}) \stackrel{\approx}{\longrightarrow}{ }^{\tau, x} G^{\prime}(\mathbb{C})
$$

for central extension $G^{\prime}$ of $G^{\text {ad }}$.

Proposition 2.7. Let ${ }^{\tau, x} \check{X}$ be the dual Hermitian symmetric space associated with $\left({ }^{\tau, x} G,{ }^{\tau, x} X\right)$. For any special point of $X$, there is a unique isomorphism (of algebraic varieties) $\varphi_{\tau, x}^{\vee}: \tau \breve{X} \rightarrow \tau, x \breve{X}$ such that

(i) the point $\tau x$ is mapped to ${ }^{2} x$, and

(ii) $\varphi_{\tau, x}^{\vee} \circ \tau(g)=(\tau, x) \circ \varphi_{\tau, x}^{\vee}$, for all $g$ in $G^{\text {ad }}(\mathbb{C})$.

Proof. The uniqueness is obvious. For the existence, note that there is a commutative diagram

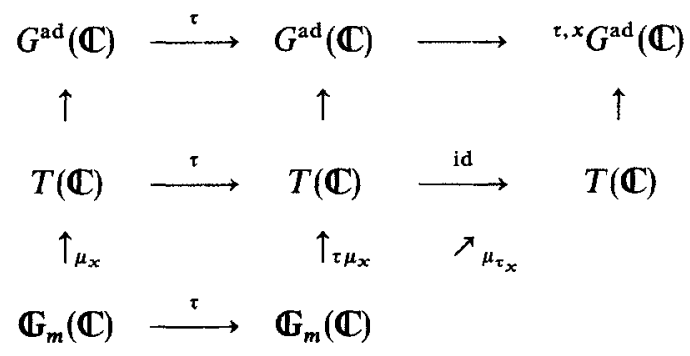

in which the unmarked arrow on the top row is $g \mapsto^{\tau, x} g$ and $T$ is a suitable subtorus of $G^{\text {ad }}$. Let $P_{x}, P^{\prime}$, and $P^{\prime \prime}$ be the subgroups of $G_{\mathbb{C}}^{\text {ad }}, G_{\mathbb{C}}^{\text {ad }}$, and ${ }^{\tau, x} G_{\mathbb{C}}^{\text {ad }}$ respectively fixing the filtrations defined by $\mu_{x}, \tau \mu_{x}$, and $\mu_{\tau_{x}}$. Then $P^{\prime}=\tau P_{x}$ and $P^{\prime \prime}$ is a subgroup of ${ }^{\tau, x} G_{\mathbb{C}}^{\text {ad }}$ such that $P^{\prime \prime}(\mathbb{C})=\left\{{ }^{\tau, x} p \mid \tau^{-1} p \in P(\mathbb{C})\right\}$. On passing to the quotients, we obtain maps $\check{X} \stackrel{\tau}{\longrightarrow} \tau \check{X} \rightarrow^{\tau, x} \check{X}$, and the second of these obviously satisfies (i) and (ii). 
Remark 2.8. (a) The subgroup $P$ of $G^{\text {ad }}$ is defined over the reflex field $E$ $=E\left(G^{\text {ad }}, X\right)$, and $\rho_{x}$ factors through $T_{E} \subset P$. The subgroup $\tau P$ of $G^{\text {ad }}$ still contains $T$ because $T$ is defined over $\mathbb{Q}$, and so we can use $\rho_{x}$ and ${ }^{t} \mathcal{G}$ to twist $\tau P$. Clearly ${ }^{\tau, x}(\tau P)=P^{\prime \prime}$.

(b) Let $x^{\prime}$ be a second special point of $X$. Then ${ }^{\tau, x} g \mapsto^{\tau, x^{\prime}} g$ is an isomorphism ${ }^{\tau, x} G^{\text {ad }}(\mathbb{C}) \rightarrow{ }^{\tau, x^{\prime}} G^{\text {ad }}(\mathbb{C})$ giving rise to a commutative diagram

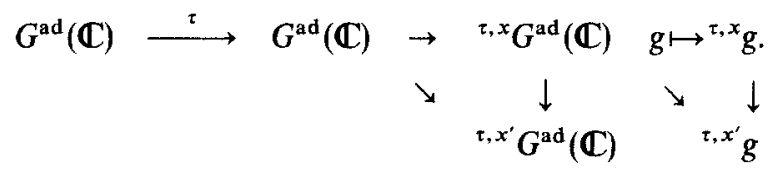

On passing to the quotients, we obtain a commutative diagram

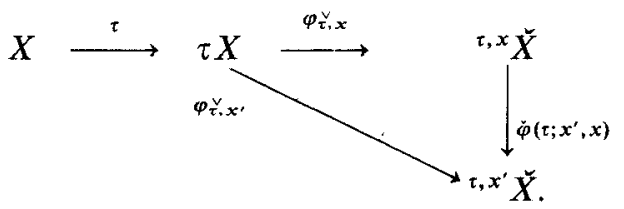

\section{The principal bundle $Y^{0}(G, X)$; statement of the first main theorem}

We begin by reviewing some elementary constructions from complex differential geometry (see for example Kobayishi and Nomizu (1963/69)).

Let $S$ be a connected complex manifold, and let $\pi_{1}=\pi_{1}(S, s)$ be the fundamental group of $S$ regarded as the group of covering transformations of the universal covering space $\widetilde{S}$ of $S$ (acting on the left). A homomorphism $r: \pi_{1}(S, s) \rightarrow G$ from $\pi_{1}(S, s)$ into a complex Lie group $G$ gives rise to a (right) principal $G$-bundle

$$
Y(r)=\sim \backslash \widetilde{S} \times G, \quad(\gamma s, r(\gamma) g) \sim(s, g)
$$

on $S$, and there is a canonical flat connection on $Y(r)$. Every principal $G$-bundle $Y$ over $S$ admitting a flat connection arises in this way.

Let $V$ be a complex vector space. A homomorphism $r: \pi_{1}(S, s) \rightarrow G L(V)$ gives rise to a vector bundle

$$
\mathscr{V}(r)=\sim \backslash \tilde{S} \times V, \quad(\gamma s, r(\gamma) v) \sim(s, v)
$$

on $S$, and there is a canonical flat connection on $\mathscr{V}$. The sections of $Y(r)$ over any open subset $U$ of $S$ can be identified with the isomorphisms $a: \mathcal{O}_{U} \otimes V \stackrel{\approx}{\longrightarrow} \mathscr{V} \mid U$ (trivializations of $\mathscr{V}$ over $U$ ).

Now suppose that $r$ factors through a reductive algebraic subgroup $G$ of $G L(V)$. There will exist a finite family of tensors $\left(t_{\alpha}\right)$ of $V$ such that $G$ is the subgroup fixing the $t_{\alpha}$. Each $t_{\alpha}$ gives rise to a global tensor $t_{\alpha}^{\prime}$ of $\mathscr{V}$, and the sections of $Y(r)$ over any open subset $U$ can then be identified with the isomorphisms $a: \mathcal{O}_{U} \otimes V \stackrel{\approx}{\longrightarrow} \mid U$ under which each $1 \otimes t_{\alpha}$ corresponds to $t_{\alpha}^{\prime} \mid U$ (trivializations of $\mathscr{V}$ over $U$ respecting the $t_{a}$ ). 
Proposition 3.1. In addition to the hypotheses in the last paragraph, assume that $S$ is algebraic, that $\mathscr{V}$ is an algebraic vector bundle on $S$, and that the tensors $t_{\alpha}^{\prime}$ are algebraic sections of the $T_{j}^{i} \mathscr{V}$. Then $Y(r)$ is algebraic, and it represents the functor of $S$-varieties whose value on $\pi: T \rightarrow S$ is the set of isomorphisms $a: \mathcal{O}_{T} \otimes V \stackrel{\approx}{\longrightarrow} \pi^{*} \mathscr{V}$ such that $1 \otimes t_{\alpha}$ corresponds to $\pi^{*}\left(t_{\alpha}^{\prime}\right)$ for all $\alpha$.

Proof. Suppose first that $G=G L(V)$ (and there are no tensors). If $\mathscr{V}$ is trivial, i.e., $\mathscr{V}=\mathcal{O}_{S} \otimes V$, then the functor is represented by $G_{S}$. Since $\mathscr{V}$ is locally trivial for the Zariski topology, it follows easily that the functor is represented by a $G_{S}$-torsor $Y$, and it is obvious from the discussion preceding the proposition that the analytic space associated with $Y$ is $Y(r)$. In the general case, the tensors $t_{\alpha}$ define a $G_{\mathbb{C}}$-subtorsor of the $G L(V)_{\mathbb{C}^{-t o r s o r}} Y$ whose associated analytic space is again $Y(r)$.

We apply these remarks to a pair $(G, X)$ defining a connected Shimura variety. For each $\Gamma \in \tilde{\Sigma}(G), \Gamma$ is the fundamental group of $S_{\Gamma}^{0}$ and (by definition) $\Gamma \subset G(\mathbb{Q}) \subset G(\mathbb{C})$. The above construction gives us a principal $G(\mathbb{C})$-bundle $Y_{\Gamma}^{0}(G, X)=\Gamma \backslash X \times G(\mathbb{C})$ over $S_{\Gamma}^{0}(G, X)$. Because of our conventions we are forced to turn this into a left principal bundle (by making $g \in G(\mathbb{C})$ act as $g^{-1}$ in the natural action; that is, $\left.g[x, c]=\left[x, c g^{-1}\right]\right)$. For varying $\Gamma$, these bundles form a projective system $Y^{0}(G, X)$, which can be regarded as a principal $G(\mathbb{C})$-bundle over $S^{0}(G, X)$. In the case that $G$ is simply connected,

$$
Y^{\circ}(G, X)=G(\mathbb{Q}) \backslash X \times G(\mathbb{C}) \times G\left(\mathbb{A}^{f}\right)
$$

with $q \in G(\mathbb{Q})$ acting on $(x, c, a) \in X \times G(\mathbb{C}) \times G\left(\mathbb{A}^{f}\right)$ according to the rule

$$
q(x, c, a)=(q x, q c, q a) .
$$

There is an action of $G^{\text {ad }}(\mathbb{Q})^{+}$on $Y^{0}(G, X): g \in G^{\text {ad }}(\mathbb{Q})^{+}$maps $[x, c]_{\Gamma} \epsilon$ $\Gamma \backslash X \times G(\mathbb{C})$ to $[\mathrm{g} x, \operatorname{ad}(\mathrm{g}) c]_{r^{\prime}} \in \Gamma^{\prime} \backslash X \times G(\mathbb{C})$, where $\Gamma^{\prime}=\operatorname{ad}(\mathrm{g}) \Gamma$. We let $G(\mathbb{Q})$ act on $S^{0}(G, X)$ through the map $G(\mathbb{Q}) \rightarrow G^{\text {ad }}(\mathbb{Q})$; this extends by continuity to an action of the closure $G(\mathbb{Q})_{+}^{-}$of $G(\mathbb{Q})_{+}$in $G\left(\mathbb{A}^{f}\right)$. Therefore $G(\mathbb{C}) \times G\left(\mathbb{Q}_{+}^{-}\right.$ and $G^{\text {ad }}(\mathbb{Q})^{+}$both act on $Y^{0}(G, X)$. If $q \in G(\mathbb{Q})_{+}^{-}$, then $(q, q) \in G(\mathbb{C}) \times G(\mathbb{Q})_{+}^{-}$and the image of $q$ in $G^{\text {ad }}(\mathbb{Q})^{+}$have the same action, and consequently we obtain an action of

$$
\mathscr{G}(G) \stackrel{d f}{=}\left(G(\mathbb{C}) \times G(\mathbb{Q})_{+}^{-}\right) *_{G(\mathbb{Q})_{+}} G^{\text {ad }}(\mathbb{Q})^{+}
$$

on $Y^{0}(G, X)$. There are obvious homomorphisms $G(\mathbb{C}) \rightarrow \mathscr{G}(G) \rightarrow G^{\text {ad }}(\mathbb{Q})^{+\wedge}$, and the action of $\mathscr{G}(G)$ on $Y^{0}(G, X)$ is compatible via these maps with the actions of $G(\mathbb{C})$ and $G^{\text {ad }}(\mathbb{Q})^{+\wedge}$ on $Y^{0}(G, X)$ and $S^{0}(G, X)$ respectively.

In the case that $G$ is simply connected, $G(\mathbb{Q})_{+}^{-}=G\left(\mathbb{A}^{f}\right)$, and the actions are given by

$$
\begin{aligned}
& a[x, z, g]=\left[x, z, g a^{-1}\right], \quad x \in X, z \in G(\mathbb{C}), a, g \in G\left(\mathbb{A}^{f}\right), \\
& c[x, z, g]=\left[x, z c^{-1}, g\right], \quad x \in X, c, z \in G(\mathbb{C}), g \in G\left(\mathbb{A}^{f}\right) .
\end{aligned}
$$

In the general case, $\mathscr{G}(G)$ is generated by the images of $G(\mathbb{C}), \widetilde{G}\left(\mathbb{A}^{f}\right)$, and $G^{\text {ad }}(\mathbb{Q})^{+}$. 
Now let $r: G_{\mathbb{C}} \rightarrow G L(V)$ be a representation of $G_{\mathbb{C}}$. In this case we obtain a vector bundle $\mathscr{V}(r)$ on $S^{0}(G, X)$ together with an action of $G(\mathbb{Q})_{-}^{+}$on $\mathscr{V}(r)$.

Proposition 3.2. The principal $G(\mathbb{C})$-bundle $Y^{\circ}(G, X)$ is algebraic, and the elements of $\mathscr{G}(G)$ act algebraically on it.

Proof. We shall need to use the following result.

Lemma 3.3. Let $S$ be an algebraic variety embedded as an open subvariety of a complete algebraic variety $\bar{S}$.

(a) If $\bar{S}-S$ has codimension $\geqq 3$, then the functor $\mathscr{F} \mapsto \mathscr{F}^{\text {an }}$ taking an algebraic vector bundle on $S$ to its associated analytic vector bundle defines an equivalence of categories.

(b) If $\bar{S}-S$ has codimension $\geqq 2$, then $\mathscr{F} \mapsto \mathscr{F}^{\text {an }}$ is fully faithful, and $\Gamma(S, \mathscr{F})$ $=\Gamma\left(S, \mathscr{F}^{\text {an }}\right)$.

Proof. This follows from theorems of Serre, Grothendieck, Siu, and Trautmann; see Hartshorne (1970), p. 222.

We first prove the proposition under the assumption that the boundary of $S_{\Gamma}^{0}(G, X)$ in its Baily-Borel compactification has codimension $\geqq 3$. Choose a faithful representation $r: G \rightarrow G L(V)$ of $G$, and let $\left(t_{\alpha}\right)$ be a finite family of tensors of $V$ such that $G$ is the subgroup of $G L(V)$ fixing the $t_{\alpha}$. Then (3.3a) shows that the sheaf $\mathscr{V}_{\Gamma}$ on $S_{\Gamma}^{0}(G, X)$ corresponding to $V$ is algebraic, and (3.3b) shows that the global tensors $t_{\alpha}^{\prime}$ are algebraic. It now follows from Proposition 3.1 that $Y_{\Gamma}^{0}(G, X)$ is algebraic. An element of $G(\mathbb{Q})_{+}^{-}$acts as an algebraic morphism on the family $\left(Y_{\Gamma}^{0}(G, X)\right)$ because (3.3a) shows that it does so on the family $\left(\mathscr{V}_{\Gamma}\right)$. On applying this to $G^{\text {ad }}$ we find that an element of $G^{\text {ad }}(\mathbb{Q})^{+}$acts algebraically on $Y_{\Gamma}^{0}\left(G^{\mathrm{ad}}, X\right)$, and therefore on $Y_{\Gamma}^{0}(G, X)$, because $Y_{\Gamma}^{0}(G, X)$ is a finite covering of $Y_{\Gamma}^{0}\left(G^{\text {ad }}, X\right)$. Finally an element of $G(\mathbb{C})$ acts algebraically on $Y^{0}(G, X)$ because it defines a morphism of the functor that $Y^{0}(G, X)$ represents.

Next we assume that the boundary has codimension $\geqq 2$. In this case, there will be a totally real field $F$ and a pair $\left(G_{*}, X_{*}\right)$ with $G_{*}=\operatorname{Res}_{F / \mathbb{Q}} G$ such that the boundary of $S_{\Gamma}^{0}\left(G_{*}, X_{*}\right)$ has codimension $\geqq 3$ and the natural map $G \hookrightarrow G_{*}$ sends $X$ into $X_{*}$. Choose a faithful representation $r_{*}$ of $G_{*}$, and let $r$ be its restriction to $G$. Then the sheaf $\mathscr{V}$ on $S^{0}(G, X)$ defined by $r$ is obtained by restriction from the similar sheaf on $S^{o}\left(G_{*}, X_{*}\right)$, and so it is algebraic. Moreover the global tensors of $\mathscr{V}$ are again algebraic, and so the same argument as before applies.

In the only remaining case, the boundary has codimension $=1$. But then $G$ is $S L_{2}$ or $P G L_{2}$, and the result is easy to prove, for example by making use of the universal elliptic curve (cf. $\S 6$ ).

In summary, with a pair $(G, X)$ defining a connected Shimura variety, we have associated a $G_{\widetilde{C}}$-torsor $Y^{0}(G, X)$ over $S^{0}(G, X)$, a flat connection $\nabla$ on $Y^{0}(G, X)$, and an action $a$ of $\mathscr{G}(G)$ on $\left(Y^{\circ}(G, X), V\right)$; moreover, a special point $x$ on $X$ defines a point $y \in Y^{0}(G, X)$ lying over $[x]$, namely, its image under the map $X \rightarrow Y^{0}(G, X)$. The association is functorial: a morphism $(G, X) \rightarrow\left(G^{\prime}, X^{\prime}\right)$ carrying a special point $x$ of $X$ to a special point $x^{\prime}$ of $X^{\prime}$ 
gives rise to a morphism of quadruples

$$
\left(Y^{0}(G, X), \nabla, a, y\right) \rightarrow\left(Y^{0}\left(G^{\prime}, X^{\prime}\right), \nabla^{\prime}, a^{\prime}, y^{\prime}\right) .
$$

We need an adèlic version of (3.1). Write $p: Y^{0}(G, X) \rightarrow S^{0}(G, X)$ for the structure map, and let $r: G \hookrightarrow G L(V)$ be a faithful representation of $G$. Let $\left(t_{\alpha}\right)$ be a finite family of tensors such that $G$ is the subgroup of $G L(V)$ fixing the the $t_{\alpha}$, and let $t_{\alpha}^{\prime}$ be the global tensor corresponding to $t_{\alpha}$. Then there is a canonical equivariant trivialization $a_{0}: \mathcal{O}_{\mathrm{Y}^{0}} \otimes V \stackrel{\approx}{\longrightarrow} p^{*} \mathscr{V}(r)$, and under the trivialization, $1 \otimes t_{\alpha}$ corresponds to $p^{*}\left(t_{\alpha}\right)$ for each $\alpha$. The next proposition will show that $Y^{0}(G, X)$ is universal for this property.

Proposition 3.4. Let $r: G \hookrightarrow G L(V),\left(t_{\alpha}\right)$, and $\left(t_{\alpha}^{\prime}\right)$ be as above. For any morphism $\pi: T \rightarrow S^{0}(G, X)$ of $\mathscr{G}(G)$-varieties and equivariant isomorphism $a: \mathcal{O}_{T} \otimes V \stackrel{\approx}{\longrightarrow} \pi^{*} \mathscr{V}(r)$ such that $1 \otimes t_{\alpha} \leftrightarrow \pi^{*}\left(t_{\alpha}^{\prime}\right)$ for all $\alpha$, there is a unique $\mathscr{G}(G)$-equivariant $S^{0}$-morphism $\psi: T \rightarrow Y^{0}(G, X)$ such that $a$ is the inverse image of $a_{0}$.

Proof. This follows easily from (3.1).

Proposition 3.5. There is a canonical $G(\mathbb{C})$-equivariant map

$$
\gamma(G, X): Y^{0}(G, X) \rightarrow \check{X} .
$$

Proof. As there is a canonical map $Y^{0}(G, X) \rightarrow Y^{0}\left(G^{\text {ad }}, X\right)$, and $\check{X}$ is unchanged when $G$ is replaced by $G^{\text {ad }}$, we may assume that $G=G^{\text {ad }}$. Let $r: G \rightarrow G L(V)$ be a faithful representation of $G$ such that the corresponding vector bundle $\mathscr{V}$ on $S^{0}(G, X)$ is algebraic, and consider the constant vector bundle $\mathscr{V}_{\Gamma}$ $=Y_{\Gamma}^{0}(G, X) \times V$. According to (3.1), an element $y \in Y_{\Gamma}^{0}$ can be identified with an isomorphism $a_{y}: V \stackrel{\approx}{\longrightarrow}\left(\mathscr{V}_{\Gamma}\right)_{y}$. Endow $\left(\mathscr{V}_{\Gamma}\right)_{y}$ with the Hodge filtration defined by the Hodge structure $a_{y} \circ{ }^{\circ} \circ h_{x}: \mathbf{S} \rightarrow G L\left(\left(\mathscr{V}_{\Gamma}\right)_{y}\right)$, where $x_{\Gamma}$ is the image of $y$ in $S_{\Gamma}^{0}(G, X)$. Then $(2.4 \mathrm{~b})$ shows that there is a unique morphism of algebraic varieties $\gamma_{\Gamma}: Y_{\Gamma}(G, X) \rightarrow \check{X}$ such that $\gamma_{\Gamma}^{*}(\mathscr{V}) \approx \mathscr{V}_{\Gamma}$ as filtered vector bundles. For varying $\Gamma$, the $\gamma_{\Gamma}$ from a projective system, that is, a morphism $\gamma: Y^{0}\left(G^{\text {ad }}, X\right) \rightarrow \check{X}$. The map commutes with the actions of $G(\mathbb{C})$.

Remark 3.6. (a) The composite of the canonical map $X \rightarrow Y^{0}(G, X)$ with $\gamma(G, X)$ is the Borel embedding $\beta$.

(b) When $G$ is simply connected, $\gamma$ is the map $[x, c, a] \mapsto c^{-1} \beta(x), x \in X$, $c \in G(\mathbb{C}), a \in G\left(\mathbb{A}^{f}\right)$.

Let $\tau$ be an automorphism of $\mathbb{C}$, and let $x$ be a special point of $X$. The point $\left(z_{\infty}(\tau), \operatorname{sp}(\tau)\right) \in^{\tau} \mathbb{S}\left(\mathbb{A}^{\prime}\right)$ defines an isomorphism

$$
g \mapsto^{\tau, x} g: G\left(\mathbb{A}^{\prime}\right) \rightarrow^{\tau, x} G\left(\mathbb{A}^{\prime}\right) .
$$

(Recall that $\mathbb{A}^{\prime}=\mathbb{C} \times \mathbb{A}^{f}$.) 
Proposition 3.7. There is a canonical map

$$
\left.g \mapsto^{\tau, x} g: \mathscr{G}(G) \rightarrow \mathscr{G}^{\mathrm{t}, x} G\right)
$$

compatible with the above map.

Proof. For simplicity, we first prove this under the assumption that $G$ is simply connected. Choose a pair $\left(G_{1}, X_{1}\right)$ as in (A.4) of the Appendix. Let $H$ be the torus $G_{1} / G$, and let $v$ be the map $G_{1} \rightarrow H$. As in (1.5), we write $H(\mathbb{R})_{+}$for $v\left(Z_{1}(\mathbb{R})\right) \subset H(\mathbb{R})$ and $H(\mathbb{Q})_{+}$for $H(\mathbb{Q}) \cap H(\mathbb{R})_{+}$. Because of (A.4c), any $q \in G^{\text {ad }}(\mathbb{Q})^{+}$lifts to an element $\tilde{q} \in G_{1}(\mathbb{Q})_{+}$, and one checks immediately that $(c, a)^{*} q \mapsto[c \tilde{q}, a \tilde{q}]$ is a well-defined homomorphism $\mathscr{G}(G) \rightarrow G_{1}\left(\mathbb{A}^{\prime}\right) / Z_{1}(\mathbb{Q})$.

Lemma 3.8. There is an exact sequence

$$
0 \rightarrow \mathscr{G}(G) \rightarrow G_{1}\left(\mathbb{A}^{\prime}\right) / Z_{1}(\mathbb{Q}) \stackrel{v}{\longrightarrow} H\left(\mathbb{A}^{\prime}\right) / H(\mathbb{Q})_{+} \rightarrow 0
$$

Proof. We first show that the final map is surjective. For this it suffices to show that $v$ defines a surjection $G_{1}\left(\mathbb{A}^{f}\right) \rightarrow H\left(\mathbb{A}^{f}\right)$. Choose $\mathbb{Z}$-structures on $G_{1}$ and $H$. Then Lang's lemma applied to the reduction of $G$ shows that $G_{1}\left(\mathbb{Z}_{p}\right) \rightarrow H\left(\mathbb{Z}_{p}\right)$ is surjective for almost all $p$. It therefore suffices to show that $G_{1}\left(\mathbb{Q}_{p}\right) \rightarrow H\left(\mathbb{Q}_{p}\right)$ is surjective for all $p$, but this follows from the fact that $H^{1}\left(\mathbb{Q}_{p}, G\right)=0$ for all finite primes $p$.

Next we prove the exactness at the middle term. From the diagram

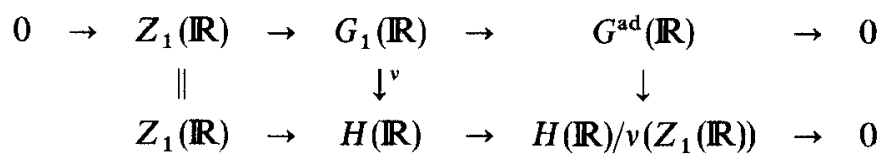

we see that $G^{\text {ad }}(\mathbb{R})^{+}$maps into the identity component of $H(\mathbb{R}) / v\left(Z_{1}(\mathbb{R})\right)$. But $H(\mathbb{R}) / v\left(Z_{1}(\mathbb{R})\right)$ is discrete, and so $G^{\text {ad }}(\mathbb{R})^{+}$maps into $v\left(Z_{1}(\mathbb{R})\right)$. It follows from this that $\mathscr{G}(G)$ maps to zero under the composite of the two maps.

Suppose that $v(c, a) \in H(\mathbb{Q})_{+} \times H(\mathbb{Q})_{+}$for some $(c, a) \in G_{1}(\mathbb{C}) \times G_{1}\left(\mathbb{A}^{f}\right)$, say $v(c, a)=(q, q)$. Consider the diagram

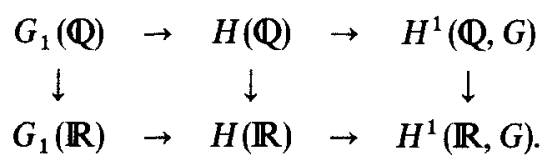

The image of $q$ in $H(\mathbb{R})$ lifts to $Z_{1}(\mathbb{R}) \subset G_{1}(\mathbb{R})$ because it lies in $H(\mathbb{R})_{+}$. Therefore the image of $q$ in $H^{1}(\mathbb{Q}, G)$ maps to zero in $H^{1}(\mathbb{R}, G)$, which implies that it is zero by the Hasse principle. Hence $q$ lifts to an element $\tilde{q}$ in $G_{1}(\mathbb{Q})$. Now $v\left(c \tilde{q}^{-1}, a \tilde{q}^{-1}\right)=1$, and so $c^{\prime} \stackrel{d f}{=} c \tilde{q}^{-1}$ and $a^{\prime} \stackrel{d f}{=} a \tilde{q}^{-1}$ lie in $G(\mathbb{C})$ and $G\left(\mathbb{A}^{f}\right)$ respectively. Let $\bar{q}$ be the image of $\tilde{q}$ in $G^{\text {ad }}(\mathbb{Q})$. Then $\left(c^{\prime}, a^{\prime}\right) * \bar{q} \in \mathscr{G}(G)$, and it maps to $(c, a) \bmod \left(Z_{1}(\mathbb{Q})\right)$.

Finally we prove the exactness at the first term. Let $(c, a) * q \in G^{\text {ad }}(\mathbb{Q})^{+\wedge}$, and choose a lifting $\tilde{q}$ of $q$ to $G_{1}(\mathbb{Q})$. If $(c, a) * q$ maps to zero in $G_{1}\left(\mathbb{A}^{\prime}\right) / Z_{1}(\mathbb{Q})$, 
then there exists a $z \in Z_{1}(\mathbb{Q})$ such that $(c \tilde{q}, a \tilde{q})=(z, z)$. Now $c$ $=z \tilde{q}^{-1} \in G(\mathbb{C}) \cap G_{1}(\mathbb{Q})=G(\mathbb{Q})$; write it $q^{\prime}$. Then $q^{\prime} \tilde{q} \in Z_{1}(\mathbb{Q})$, and so the image of $q^{\prime}$ in $G^{\text {ad }}(\mathbb{Q})$ is $q^{-1}$. Consequently, $\left(q^{\prime}, q^{\prime}\right) * q=1$, but $\left(q^{\prime}, q^{\prime}\right) * q=(c, a) * q$.

We now return to the proof of the proposition. If $z \in Z_{1}(\Phi)$ then the element $[s \cdot z]$ of ${ }^{\tau, x} G_{1}(\Phi)$ is independent of $s$. Therefore $z \mapsto[s \cdot z]$ is an inclusion $Z \hookrightarrow^{r, x} G_{1}$, and the map

$$
g \mapsto^{\tau, x} g=\left[\left(z_{\infty}(\tau), \operatorname{sp}(\tau)\right) \cdot g\right]: G_{1}\left(\mathbb{A}^{\prime}\right) \rightarrow^{\tau, x} G_{1}\left(\mathbb{A}^{\prime}\right)
$$

induces the identity map on $Z_{1}\left(\mathbb{A}^{\prime}\right)$. In particular it maps $Z_{1}(\mathbb{Q}) \subset G_{1}\left(\mathbb{A}^{\prime}\right)$ into $Z_{1}(\mathbb{Q}) \subset{ }^{\tau, x} G_{1}\left(\mathbb{A}^{\prime}\right)$, and so we have a map $\psi: G_{1}\left(\mathbb{A}^{\prime}\right) / Z_{1}(\mathbb{Q}) \rightarrow^{\tau, x} G_{1}\left(\mathbb{A}^{\prime}\right) / Z_{1}(\mathbb{Q})$. Since $g \mapsto^{\tau, x} g: G_{1}\left(\mathbb{A}^{\prime}\right) \rightarrow^{\tau, x} G_{1}\left(\mathbb{A}^{\prime}\right)$ induces the identity map on $H\left(\mathbb{A}^{\prime}\right), \psi$ maps $\mathscr{G}(G)$ into $\mathscr{G}\left({ }^{\tau, x} G\right)$, and this is the map we want.

When $G$ is not simply connected, then $\mathscr{G}(G)$ is the quotient of $\mathscr{G}(\tilde{G})$ by

$$
\mathscr{G}(\tilde{G}, G) \stackrel{d f}{=} \operatorname{Ker}\left(\tilde{Z}\left(\mathbb{A}^{\prime}\right) / \tilde{Z}(\mathbb{Q}) \rightarrow Z\left(\mathbb{A}^{\prime}\right) / Z(\mathbb{Q})\right),
$$

where $\tilde{Z}$ and $Z$ are the centres of $\widetilde{G}$ and $G$ respectively. There is a canonical isomorphism $\mathscr{G}(\tilde{G}, G) \stackrel{\approx}{\longrightarrow} \mathscr{G}\left({ }^{\tau, x} \widetilde{G},{ }^{\tau, x} G\right)$ and $g \mapsto^{\tau, x} g: \mathscr{G}(\widetilde{G}) \rightarrow \mathscr{G}\left({ }^{\tau, x} \widetilde{G}\right)$ is compatible with this isomorphism (cf. the above discussion involving $Z_{1}$ ). Hence the map $g \mapsto^{r, x} G$ on $\mathscr{G}(G)$ can be defined by passing to the quotient with the map $g \mapsto^{\tau, x} g$ on $\mathscr{G}(\tilde{G})$.

From the pair $\left({ }^{\tau, x} G,{ }^{\tau, x} X\right)$ and the special point ${ }^{\tau} x$ of ${ }^{\tau, x} X$, we can construct a ${ }^{\tau, x} G(\mathbb{C})$-torsor $Y^{0}\left({ }^{\tau, x} G,{ }^{\tau, x} X\right)$, a flat connection ${ }^{\tau, x} V$, an action ${ }^{\tau, x} a$ of $\mathscr{G}(G)$ on $\left(Y^{0}\left({ }^{\tau, x} G,{ }^{\tau, x} X\right),{ }^{\tau, x} V\right)$, and a point ${ }^{\tau} y$ on $Y^{0}\left({ }^{\tau, x} G,{ }^{\tau, x} X\right)$.

Proposition 3.9. Let $x^{\prime}$ be a second special point. There is a canonical isomorphism $\varphi^{Y}\left(\tau ; x^{\prime}, x\right): Y^{0}\left({ }^{\tau, x} G,{ }^{\tau, x} X\right) \rightarrow Y^{0}\left({ }^{\tau, x} x^{\prime} G,{ }^{\tau, x^{\prime}} X\right)$ such that

(i) $\varphi^{Y}\left(\tau ; x^{\prime}, x\right)$ is compatible with the flat connections;

(ii) $\varphi^{Y}\left(\tau ; x^{\prime}, x\right) \circ\left({ }^{\tau, x} g\right)=\left(\tau, x^{\prime} g\right) \circ \varphi^{Y}\left(\tau ; x^{\prime}, x\right)$ for all $g \in \mathscr{G}(G)$;

(iii) the following diagram commutes

$$
\begin{array}{cccc}
\varphi^{\vee}\left(\tau ; x^{\prime}, x\right): & { }^{\tau, x} \check{X} & \rightarrow & \uparrow^{\tau, x^{\prime}} \check{X} \\
& \uparrow^{\gamma} & & \uparrow^{\gamma} \\
\varphi^{Y}\left(\tau ; x^{\prime}, x\right): & Y^{0}\left({ }^{\tau, x} G,{ }^{\tau, x} X\right) & \rightarrow & Y^{0}\left({ }^{\tau, x} x^{\prime} G,{ }^{\tau, x^{\prime}} X\right) \\
& \downarrow & & \downarrow \\
\varphi^{0}\left(\tau ; x^{\prime}, x\right): & S^{0}\left({ }^{\tau, x} G,{ }^{\tau, x} X\right) & \rightarrow & S^{0}\left(^{\tau, x^{\prime}} G,{ }^{\tau, x^{\prime}} X\right) .
\end{array}
$$

Proof. The proof of (1.3) applies with minor modifications. For simplicity, we first assume that $G$ is simply connected. Choose a pair $\left(G_{1}, X_{1}\right)$ (with the weight equal to zero) and an isomorphism $t: \rho_{x_{1} *}\left({ }^{\mathrm{r}} \Xi\right) \stackrel{\approx}{\longrightarrow} \rho_{x_{1}^{\prime}}\left({ }^{\mathrm{I}} \mathrm{S}\right)$, as in the proof of (1.3). Again $t$ defines an isomorphism $f_{1}:{ }^{\tau, x} G_{1} \stackrel{\approx}{\longrightarrow}{ }^{\tau, x^{\prime}} G_{1}$. Let $s$ and $s^{\prime}$ be the images of $\left(z_{\infty}(\tau), \operatorname{sp}(\tau)\right)$ in $\rho_{x_{1} *}\left({ }^{\tau} \mathbb{S}\right)\left(\mathbb{A}^{\prime}\right)$ and $\rho_{x_{1}^{\prime} *}\left({ }^{\top} \mathbb{S}\right)\left(\mathbb{A}^{\prime}\right)$ respectively. Then there is an element $\gamma_{1} \in{ }^{\tau, x} G_{1}\left(\mathbb{A}^{\prime}\right)$ such that $t_{1}\left(s \gamma_{1}\right)=s^{\prime}$. The same argument as in the proof of $(1.3)$ shows that the image of $\gamma_{1}$ in $H\left(\mathbb{A}^{\prime}\right)$ lies in its subgroup $H(\mathbb{Q})$, and the Remark 1.5 shows that it lies in $H(\mathbb{Q})_{+} \subset H(\mathbb{Q})$. Therefore, accord- 
ing to (3.8), the image $\gamma$ of $\gamma_{1}$ in $\left.{ }^{\tau, x} G_{1}\left(\mathbb{A}^{\prime}\right)\right|^{\tau, x} Z_{1}(\mathbb{Q})$ lies in $\left.\mathscr{G}^{\tau, x} G\right)$. Now $\varphi^{0}\left(\tau ; x^{\prime}, x\right)$ can be defined to be $Y^{0}(f) \circ(\gamma)$ where $f$ is the restriction of $f_{1}$ to ${ }^{\tau, x} G$.

In the case that $G$ is not simply connected, first construct the diagram for $(\tilde{G}, X)$, and then pass to the appropriate quotients.

Theorem 3.10. Let $(G, X)$ be a pair defining a connected Shimura variety, and let $x$ be a special point of $X$. For any automorphism $\tau$ of $\mathbb{C}$, there is a unique isomorphism $\varphi_{\tau, x}^{Y}: \tau Y^{0}(G, X) \rightarrow Y^{0}\left({ }^{\tau, x} G,{ }^{\tau, x} X\right)$ lying over $\varphi_{\tau, x}^{0}$ and such that

(i) $\tau y$ is mapped to ${ }^{\tau} y$;

(ii) $\varphi_{\tau, x}^{Y} \circ \tau(g)=\left(^{\tau, x} g\right) \circ \varphi_{\tau, x}^{Y}$ for all $g \in \mathscr{G}(G)$.

Moreover,

(iii) $\varphi_{\tau, x}^{Y}$ is compatible with the flat connections on $\tau Y^{0}(G, X)$ and $Y^{0}\left({ }^{\tau, x} G,{ }^{\tau, x} X\right)$. If $x^{\prime}$ is a second special point, then $\varphi^{Y}\left(\tau ; x^{\prime}, x\right) \circ \varphi_{\tau, x}^{Y}=\varphi_{\tau, x^{\prime}}^{Y}$.

Proof. We prove only the uniqueness of $\varphi_{\tau, x}^{Y}$ here; the proof of the existence will occupy $\S 6-\S 9$.

Let $\varphi_{1}$ and $\varphi_{2}$ be two maps $\tau Y^{0}(G, X) \rightarrow Y^{0}\left({ }^{\tau, x} G,{ }^{\tau, x} X\right)$ satisfying the conditions (i) and (ii), and let $Z$ be the closed subset of $\tau Y^{0}(G, X)$ on which the two maps agree. Then (ii) shows that $Z$ contains a fibre of the map $\pi: \tau Y^{0}(G, X) \rightarrow \tau S^{0}(G, X)$ whenever it contains a single point of the fibre. Therefore $Z=\pi^{-1}\left(Z^{\prime}\right)$ for some subset $Z^{\prime}$ of $\tau S^{0}(G, X)$, and because $Z$ is closed, so also must be $Z^{\prime}$. Condition (i) shows that $Z^{\prime}$ contains $\tau[x]$, and (ii) then shows that it contains all translate of $\tau[x]$ by elements of $G^{\text {ad }}(\mathbb{Q})^{+\wedge}$. As these points make up a dense subset of $S^{0}(G, X)$ for the Zariski topology, it follows that $Z^{\prime}=S^{0}(G, X)$ and $Z=Y^{0}(G, X)$.

Corollary 3.11. Assume that there is a map $\varphi_{\tau, x}^{Y}$ as in the theorem; then there a commutative diagram:

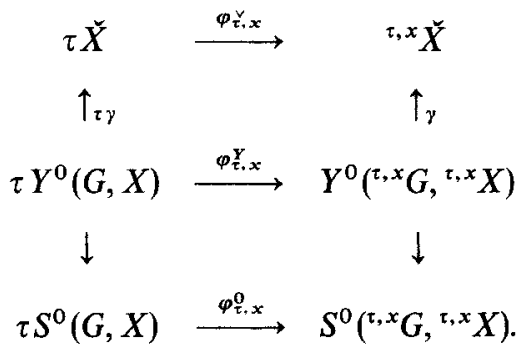

The two upper maps are compatible with the map $g \mapsto^{\tau, x} g: G(\mathbb{C}) \rightarrow^{\tau, x} G(\mathbb{C})$.

Proof. It remains to prove that the upper square commutes, i.e., that the maps $\varphi_{t, x}^{\vee} \circ \tau \gamma(G, X)$ and $\gamma\left({ }^{\tau, x} G,{ }^{\tau, x} X\right) \circ \varphi_{\tau, x}^{Y}$ are equal. They are maps $\tau Y^{0}(G, X) \rightarrow{ }^{\tau, x} \check{X}$, and we know that they agree at $\tau y$. Both maps are constant on the orbits of $G(\mathbb{Q})_{+}^{-}$in $\tau Y^{0}(G, X)$, and so they agree on all translates of $y$. Finally, both maps are compatible with the map $g \mapsto^{\tau, x} g: G(\mathbb{C}) \rightarrow^{\tau, x} G(\mathbb{C})$, and so they agree on a fibre of $\pi: \tau Y^{0}(G, X) \rightarrow \tau S^{\circ}(G, X)$ when they agree at a single point. The argument in the preceding proof now shows that the closed subset where the maps agree is the whole of $\tau Y^{0}(G, X)$. 


\section{Automorphic vector bundles}

In this section we define automorphic vector bundles, and discuss some of their structure. For a similar discussion in the case of nonconnected Shimura varieties, see Harris (1985), § 3.

Let $(G, X)$ be a pair defining a connected Shimura variety, and let $\beta: X \hookrightarrow \check{X}$ be the Borel embedding. The action of $G(\mathbb{R})_{+}$on $X$ extends to a transitive action of $G(\mathbb{C})$ on $\breve{X}$. Since $\check{X}$ is a projective algebraic variety, every holomorphic vector bundle on $\check{X}$ is algebraic. By a $G_{\mathbb{C}^{-}}$vector bundle on $\check{X}$, we mean a vector bundle $(\mathscr{J}, p)$ on $\check{X}$ together with an action of $G_{\mathbb{C}}$ on $\mathscr{J}$ (as an algebraic variety) such that

(a) $p(g \cdot w)=g \cdot p(w)$ for all $g \in G(\mathbb{C}), w \in \mathscr{J}$;

(b) the maps $g: \mathscr{J}_{x} \rightarrow \mathscr{J}_{g x}$ are linear for all $g \in G(\mathbb{C})$ and $x \in \check{X}$.

Such a vector bundle restricts to a $G(\mathbb{R})_{+}$-vector bundle $\tilde{\mathscr{V}}=\beta^{*} \mathscr{J}$ on $X$, and for each $\Gamma \in \tilde{\Sigma}(G), \mathscr{V}_{\Gamma} \stackrel{d f}{=} \Gamma \backslash \tilde{\mathscr{V}}$ is a vector bundle on $\Gamma \backslash X=S_{\Gamma}^{0}(G, X)$. The family $\mathscr{V}=\left(\mathscr{V}_{\Gamma}\right)$ forms a projective system, and there is a natural action of $G(\mathbb{Q})_{+}$ on $\mathscr{V}$

$$
g: \Gamma \backslash \tilde{\mathscr{V}} \rightarrow g \Gamma g^{-1} \backslash \tilde{\mathscr{V}}, \quad v(\bmod \Gamma) \mapsto g v\left(\bmod g \Gamma g^{-1}\right),
$$

which extends by continuity to the closure $G\left(\mathbb{Q}_{+}^{-}\right.$of $G(\mathbb{Q})_{+}$in $G\left(\mathbb{A}^{f}\right)$. A $G(\mathbb{Q})_{+}^{-}$-vector bundle $\mathscr{V}$ on $S^{0}(G, X)$ arising in this way from a $G(\mathbb{C})$-vector bundle $\mathscr{J}$ on $\check{X}$ will be called an automorphic vector bundle. We sometimes write $\mathscr{V}=\mathscr{V}(\mathscr{J})$. For each $x$ in $X$, the fibre $\left(\mathscr{V}_{\Gamma}\right)_{x_{\Gamma}}=\tilde{\mathscr{V}}_{x}=\mathscr{J}_{\beta(x)}$, and so $\mathscr{V}_{[x]} \stackrel{d f}{=} \underset{\leftarrow}{\lim }\left(\mathscr{V}_{\Gamma}\right)_{x_{\Gamma}}=\mathscr{J}_{\beta(x)}$.

When $G$ is simply connected, $\mathscr{V}$ is the $G\left(\mathbb{A}^{f}\right)$-vector bundle

$$
\mathscr{V}=G(\mathbb{Q}) \backslash \tilde{\mathscr{V}} \times G\left(\mathbb{A}^{f}\right)
$$

on $S^{0}(G, X)=G(\mathbb{Q}) \backslash X \times G\left(\mathbb{A}^{f}\right)$, and the action of $a \in G\left(\mathbb{A}^{f}\right)$ is given by

$$
a[v, g]=\left[v, g a^{-1}\right] .
$$

On the other hand, when the action of $G(\mathbb{C})$ on $\mathscr{J}$ factors through $G^{\text {ad }}(\mathbb{C})$, then we can regard $\mathscr{V}$ as being the projective system $(\Gamma \backslash \tilde{\mathscr{V}}), \Gamma \in \Sigma(G)$, and the action of $G^{\text {ad }}(\mathbb{Q}) \hat{+}$ on $S^{0}(G, X)$ lifts to $\mathscr{J}$.

We wish to show that automorphic vector bundles are algebraic, but first we need a lemma.

Lemma 4.1. Let $G$ be an affine algebraic group over a field $k$, and let $\pi: Y \rightarrow S$ be a torsor for $G_{S}$ over an algebraic variety $S$.

(a) The functor $\mathscr{V} \mapsto \pi^{*} \mathscr{V}$ defines an equivalence between the category of vector bundles on $S$ and the category of G-vector bundles on $Y$.

(b) If $Y$ has a flat $G$-connection, then to give a (flat) connection on $\mathscr{V}$ is the same as to give a (flat) G-connection on $\pi^{*} \mathscr{V}$. 
Proof. (a) This follows from descent theory: the map $\pi$ is faithfully flat, and because $Y$ is a $G$-torsor, to give a descent datum on an $\mathscr{O}_{Y}$-module $\mathscr{V}^{\prime}$ is the same as to give a $G$-action on it. That $\pi^{*}(\mathscr{V})$ is locally free if and only if $\mathscr{V}$ is locally free follows from the fact that a coherent module is locally free if and only if it is flat.

(b) Again this is a straightforward application of descent theory.

Proposition 4.2. The vector bundle $\mathscr{V}(\mathscr{J})$ is obtained by descent from $\gamma^{*}(\mathscr{J})$; hence $\mathscr{V}$ and the action of $G(\mathbb{Q})_{+}^{-}$on it are algebraic.

Proof. The first statement is obvious from the commutative diagram:

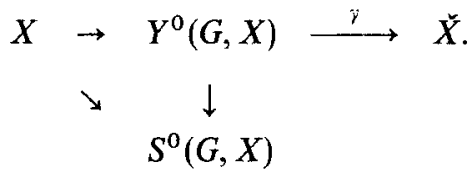

The second statement follows from the first (because of (3.2)).

Obviously, a section of $\mathscr{V}_{\Gamma}$ over $S_{\Gamma}^{0}$ gives rise to a section of $\tilde{\mathscr{V}}$ over $X$. We define an automorphic form of type $\mathscr{J}$ and level $\Gamma$ to be a section of (the analytic vector bundle) $\tilde{\mathscr{V}}$ over $X$ that arises from a section of (the algebraic vector bundle) $\mathscr{V}_{r}$. Thus an automorphic form of type $\mathscr{J}$ and any level is an element of the union $\cup \Gamma\left(S_{\Gamma}^{0}, \mathscr{V}_{\Gamma}\right)^{\prime}$, where $\Gamma\left(S_{\Gamma}^{0}, \mathscr{V}_{\Gamma}\right)^{\prime}$ denotes the image of $\Gamma\left(S_{\Gamma}^{0}, \mathscr{V}_{\Gamma}\right)$ in $\Gamma(X, \tilde{V})$. When $G^{\text {ad }}$ has no $Q$-rational factors isomorphic to $P G L_{2}$, then the boundary of $S_{T}^{0}$ in its Baily-Borel compactification has codimension $\geqq 2$, and so $\Gamma\left(S_{\Gamma}^{0}, \mathscr{V}_{\Gamma}\right)=\Gamma\left(S_{\Gamma}^{0}, \mathscr{V}_{\Gamma}^{\text {an }}\right)$ (see 3.3). Therefore, in this case, an automorphic form of type $\mathscr{J}$ and level $\Gamma$ is simply a holomorphic section of $\tilde{V}$ over $X$ fixed under the action of $\Gamma$.

Let $o$ be a point of $X$, and regard it also as a point of $\check{X}$. The isotropy group at $o$ in $G_{\mathbb{C}}$ is a parabolic subgroup $P_{0}$ of $G_{\mathbb{C}}$, and the action of $G_{\mathbb{C}}$ on $\mathscr{J}$ induces a linear action of $P_{0}$ on the fibre $\mathscr{J}_{0}$ of $\mathscr{J}$.

Proposition 4.3. The map $\mathscr{J} \mapsto \mathscr{J}_{0}$ defines an equivalence between the category of $\mathrm{G}_{\mathbb{C}}$-vector bundles $\mathscr{J}$ on $\check{X}$ and $\operatorname{Rep}_{\mathbb{C}}\left(P_{0}\right)$.

Proof. This is standard; the vector bundle corresponding to a representation $(r, V)$ of $P_{0}$ is $\mathscr{J}=G_{\mathbb{C}} \times V / P_{0}$.

Remark 4.4. Let $V=\mathscr{J}_{0}$. If $r: P_{0} \rightarrow G L(V)$ extends to a representation $(r, V)$ of $G$, then the map

$$
(g, v) \mapsto\left[g, r(g)^{-1} v\right]: G_{\mathbb{C}} \times V \rightarrow \mathscr{J}
$$

induces an isomorphism $\check{X} \times V \stackrel{\approx}{\longrightarrow}$, and so $\mathscr{V}(\mathscr{J})$ is the vector bundle associated with $(r, V)$ (as in $\S 3$ ).

Recall (2.6) that there is an equivariant commutative diagram

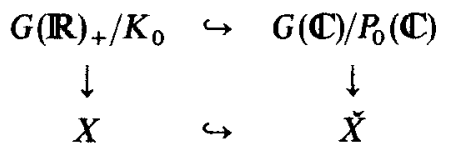


with $K_{0}$ a maximal compact subgroup of $G(\mathbb{R})_{+}$. If we let $R_{u} P_{0}$ denote the unipotent radical of $P_{0}$, then $K_{0} \hookrightarrow P_{0}$ defines an isomorphism $\left(K_{0}\right)_{\mathbb{C}} \stackrel{\approx}{\longrightarrow} P_{0} / R_{u} P_{0}$. Now (4.3) has the following corollary.

Corollary 4.5. There are natural one-to-one correspondences between isomorphism classes of the following objects:

(a) $G_{\mathbb{C}^{-v e c t o r ~ b u n d l e s ~}} \mathscr{J}_{\text {such }}$ that $R_{u} P_{0}$ acts trivially on $\mathscr{J}_{0}$;

(b) semisimple complex representations of $P_{0}$;

(c) representations of $K_{0 \mathbb{C}}$ on complex vector spaces;

(d) representations of $K_{0}$ on complex vector spaces.

Proof. A representation of $P_{0}$ is semisimple if and only if it is trivial on $R_{u} P_{0}$. Therefore the correspondence between (a) and (b) follows from (4.3). We saw above that $K_{0 \mathbb{C}}=P_{0} / R_{u} P_{0}$, which proves the correspondence between the objects in (c) and those in (a) and (b). Since $K_{0}$ is the compact form of $K_{0 \mathbb{C}}$, the correspondence between (c) and (d) is part of Weyl's unitary trick.

In the next two remarks we show that certain automorphic vector bundles have additional structures.

Remark 4.6. Suppose that $\mathscr{J}$ is a $G_{\mathbb{C}}^{\text {ad }}$-vector bundle on $\check{X}$ (rather than a $G_{\mathbb{C}^{-}}$-vector bundle). Then at each point $\left[P_{x}, \mu_{x}\right]$ of $\check{X}, \mu_{x}$ defines a filtration on the fibre $\mathscr{F}_{x}$. These filtrations vary holomorphically and define a filtration of $F^{*} \mathscr{J}$ of $\mathscr{F}$ by $G(\mathbb{C})$-stable subbundles,

$$
\ldots F^{p} \mathscr{J} \supset F^{p+1} \mathscr{J} \ldots
$$

For each $p, F^{p} \mathscr{J}$ is the $G^{\text {ad }}(\mathbb{C})$-vector bundle defined by the subrepresentation of $P_{0}$ on $\left(F^{p} \mathscr{J}\right)_{0}$. This filtration induces a holomorphic filtration of $\tilde{V}$ by $G^{\text {ad }}(\mathbb{R})^{+}$-stable subbundles, and a filtration of $\mathscr{V}$ by $G^{\text {ad }}(\mathbb{Q})^{+\wedge}$-stable algebraic subbundles $F^{p} \mathscr{V}$. For each $x$ in $X$, the action of $h_{x}$ on $\widetilde{\mathscr{V}}_{x}$ defines a splitting of the filtration,

$$
F^{p} \widetilde{\mathscr{V}}_{x}=\oplus(\tilde{\mathscr{V}})^{p^{\prime}, q^{\prime}}, \quad(\tilde{\mathscr{V}})^{p^{\prime}, q^{\prime}}=\left\{v \mid h(z) v=z^{-p^{\prime}} \bar{z}^{-q^{\prime}} v\right\},
$$

and these splittings define a decomposition of the $C^{\infty}$-bundle $\tilde{\mathscr{V}}^{\infty} \stackrel{d f}{=} \mathcal{O}_{X^{\infty}} \otimes \mathscr{V}$,

$$
\tilde{\mathscr{V}}^{\infty} \stackrel{\approx}{\longrightarrow} \oplus(\tilde{\mathscr{V}})^{p, q} .
$$

(The splitting is $C^{\infty}$ because $(\tilde{\mathscr{V}})^{p, q}=F^{p} \cap \bar{F}^{q}$, and the filtration $\bar{F}^{*}$ is anti-holomorphic.)

Remark 4.7. In the case that $\mathscr{J}$ arises from a representation of $G$ (rather than of $P_{0}$ ), it is possible to define local systems underlying the automorphic vector bundles. Before explaining this, we recall the correspondence between local systems and vector bundles with flat connection (see for example Deligne (1970), I.2). Let $S$ be a connected complex manifold and consider the tensor category of pairs $(\mathscr{V}, \nabla)$ with $\mathscr{V}$ an analytic vector bundle on $S$ and $\nabla$ a flat connection. The map $(\mathscr{V}, \nabla) \mapsto \mathbf{V} \stackrel{d f}{=} \mathscr{V}^{\nabla}$ defines an equivalence between this category and 
the tensor category of local systems of complex vector spaces on the manifold; it has quasi-inverse $\mathbf{V} \mapsto\left(\mathcal{O}_{S} \otimes_{\mathbb{C}} \mathbf{V}, \nabla_{\text {can }}\right)$.

A complex representation $r: G_{\mathbb{C}} \rightarrow G L(V)$ of $G$ defines a $G(\mathbb{C})$-vector bundle $\mathscr{I}=V \times \check{X}$ over $\check{X}$ with a canonical flat connection $V$. The flat connection defines a similar connection on $\tilde{V}=\beta^{*} \mathscr{J}(=V \times X)$ and on the automorphic vector bundle $\mathscr{V}$. In particular, we get a local system of complex vector spaces $\mathbf{V}\left(=\mathscr{V}^{\nabla}\right)$ on $S^{0}(G, X)$, stable under the action of $G(\mathbb{Q})_{+}^{-}$on $\mathscr{V}$, and such that $\mathcal{O}_{S^{o}} \otimes_{\mathbb{C}} V=\mathscr{V}$.

Suppose $\mathscr{J}$ is defined by a real representation $(r, V)$ of $G_{\mathbf{R}}$. Then for each $\Gamma \in \tilde{\Sigma}(G, X)$, the representation $(r \mid \Gamma, V)$ defines a local system of real vector spaces $\mathbf{V}_{\Gamma}$ on $\Gamma \backslash X$ such that $\mathcal{O} \otimes_{\mathbb{R}} \mathbf{V}_{\Gamma}=\mathscr{V}^{\nabla}$. The action of $G(\mathbb{Q})_{+}^{-}$on $S^{0}(G, X)$ lifts to an action on the projective system $\mathbf{V}=\left(\mathbf{V}_{\Gamma}\right)$. When $r$ factors through $G^{\text {ad }}$, the constant sheaf defined by $V$ on $X$ is (in a natural way) a polarizable variation of real Hodge structures spaces (see (2.5)), and so $\mathbf{V}$ acquires a similar structure.

Finally if $\mathscr{J}$ arises from a representation of $G^{\text {ad }}$ on a $Q$-vector space $V$, then we get a variation of rational Hodge structures $\mathbf{V}$ on $S^{\circ}(G, X)$ such that $\mathbf{V} \otimes \mathbb{C}=\mathscr{V}^{\nabla}$. In this case we can also define, for each prime $l$, a local system of $\mathbb{Q}_{l}$-vector spaces $\mathbf{V}_{l}=\left(\mathbf{V}_{l, L}\right)$ for the étale topology: $\mathbf{V}_{l, \Gamma}$ is the locally constant sheaf on $S_{\Gamma}^{0}$ associated with the representation of $\Gamma$ on $\mathbb{Q}_{l} \otimes V$ (see for example Milne (1980), p. 165). Symbolically, we may write $\mathbf{V}_{l}=\mathbb{Q}_{l} \otimes \mathbf{V}$. There is again a natural action of $G^{\text {ad }}(\mathbb{Q})^{+\wedge}$ on $\mathbf{V}$ and $\mathbf{V}_{l}$.

Remark 4.8. The construction of $\mathscr{V}$ (and the extra structure on it) is functorial in $(G, X, \mathscr{F})$. Moreover, if $\mathscr{J}$ and $\mathscr{J}^{\prime}$ are two $G_{\mathbb{C}}$-equivariant vector bundles on $\check{X}$, then an equivariant differential operator $\delta: \mathscr{J} \rightarrow \mathscr{J}^{\prime}$ gives rise (in a canonical way) to an equivariant differential operator $\gamma: \mathscr{V} \rightarrow \mathscr{V}$. (See Grothendieck (1967), IV.16.8) for the definition and basic formalism of differential operators on sheaves of $\mathcal{O}_{S}$-modules for $S$ a scheme).

Remark 4.9. We outline the relation between the above definition of automorphic forms and the more usual definition in terms of automorphy factors.

Fix a point $o \in X$, and let $\tilde{\mathscr{V}}$ be the $G(\mathbb{R})_{+}$-vector bundle on $X$ defined by a $G_{\mathbb{C}}$-vector bundle $\mathscr{J}$ on $\check{X}$. Choose a trivialization $\alpha: V \times X-\approx \widetilde{V}$, and write $\gamma(\alpha(v, x))=\alpha(j(\gamma, x) v, \gamma x)$ for $\gamma \in G(\mathbb{R})_{+}, v \in V$, and $x \in X$. Then $j: G(\mathbb{R})_{+} \times X \rightarrow G L(V)$ is a holomorphic automorphy factor for $(G, X)$ with values in $V$, and an automorphic form of level $\Gamma$ and type $\mathscr{J}$ can be identified (through $\alpha$ ) with an automorphic form of level $\Gamma$ for $j$.

Conversely, let $j$ be a holomorphic automorphy factor for $(G, X)$ with values in $V$. The map $r: K_{0} \rightarrow G L(V), k \mapsto j(k, o)$, is a representation of $K$ on $V$, which (see 4.5) defines an automorphic vector bundle $\mathscr{J}$ on $\breve{X}$. If $r$ is irreducible, then it is known that automorphic forms of level $\Gamma$ for $j$ correspond to automorphic forms of level $\Gamma$ and type $\mathscr{J}$ (see Murakami (1966), p. 137).

\section{Conjugates of automorphic vector bundles}

In this section, we state the main theorem for automorphic vector bundles and show how to deduce it from Theorem 3.10. 
Let $\mathscr{J}$ be a $G_{\mathbb{C}}$-vector bundle on $\check{X}$, and fix a special point $x$ of $X$. The $G_{\mathbb{C}^{-v e c t o r}}$ bundle $\tau \mathscr{J}$ on $\tau \check{X}$ corresponds under the isomorphism $\varphi_{\tau, x}^{\vee}: \tau \check{X} \rightarrow{ }^{\tau, x} \breve{X}$ of (2.7) to a ${ }^{\tau, x} G_{\mathbb{X}^{-}}$-vector bundle ${ }^{\tau, x} \mathscr{J}$ on ${ }^{\tau, x} \check{X}$, and ${ }^{\tau, x} \mathscr{J}$ defines an automorphic vector bundle ${ }^{\tau, x} \mathscr{V}$ on $S^{0}\left({ }^{\tau, x} G,{ }^{\tau, x} X\right)$.

Lemma 5.1. If $x^{\prime}$ is a second special point of $X$, then there is a canonical isomorphism $\varphi^{\mathscr{V}}\left(\tau ; x^{\prime}, x\right):{ }^{\tau, x} \mathscr{V} \rightarrow^{\tau, x^{\prime}} \mathscr{V}$ lying over $\varphi^{0}\left(\tau ; x^{\prime}, x\right)$ and such that

$$
\varphi^{\mathscr{V}}\left(\tau ; x^{\prime}, x\right) \circ\left(^{\tau, x} g\right)=\left(^{\tau, x} g\right) \circ \varphi^{\mathscr{V}}\left(\tau ; x^{\prime}, x\right), \quad \text { all } g \in^{\tau, x} \tilde{G}\left(\mathbb{A}^{f}\right) \text {. }
$$

Proof. From the commutative diagram in (2.8) we see that there is a canonical isomorphism $^{\tau, x} \mathscr{J} \varlimsup^{\tau, x^{\prime}} \mathscr{J}$ lying over $\check{\varphi}\left(\tau ; x^{\prime}, x\right)::^{\tau, x} \breve{X} \rightarrow^{\tau, x^{\prime}} \check{X}$, and $(4.2)$ and (3.9) show that this gives rise to the required isomorphism.

Theorem 5.2. Let $\mathscr{V}$ be an automorphic vector bundle on $S^{0}(G, X)$. There is a canonical isomorphism $\varphi_{\tau, x}^{\mathscr{V}}: \tau \mathscr{V} \rightarrow^{\tau, x} \mathscr{V}$ such that

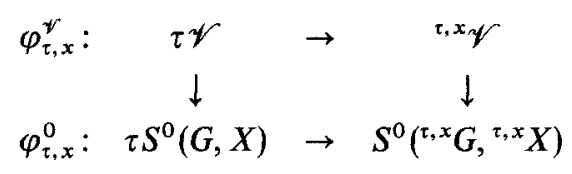

commutes and $\varphi_{\tau, x}^{\vee} \circ \tau(g)=\left(^{\mathfrak{\tau}, x} g\right) \circ \varphi_{\tau, x}^{\mathscr{V}}$ for all $g \in G(\mathbb{Q})_{+}^{-} ;$moreover, if $x^{\prime}$ is a second special point of $X$, then $\varphi^{\mathscr{V}}\left(\tau ; x^{\prime}, x\right) \circ \varphi_{\tau, x}^{\mathscr{V}}=\varphi_{\tau, x^{\prime}}^{\mathscr{V}}$.

Proof. From (3.11) we have a commutative diagram

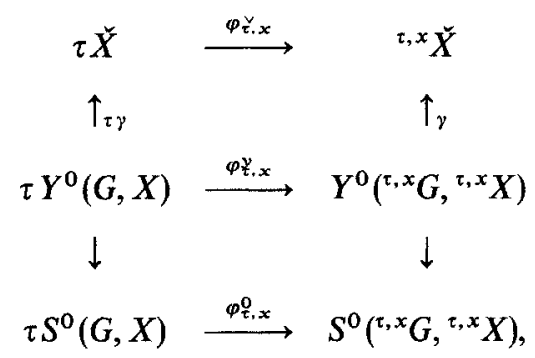

and from the very definition of ${ }^{\tau, x} \mathscr{J}$, there is a commutative diagram

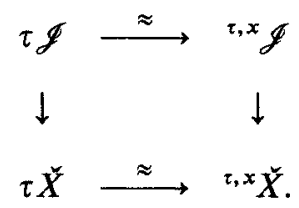

On pulling this back, we obtain a similar diagram

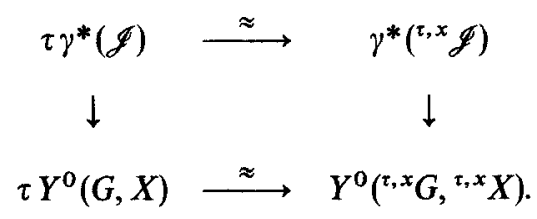


Now (4.2) proves that this gives rise to canonical commutative diagram as in the statement of the theorem. Moreover it is clear from (3.10(ii)) that the map $\varphi_{\tau, x}^{t}$ commutes with the actions of the Hecke operators, and it follows from (3.11) that $\varphi^{ף}\left(\tau ; x^{\prime}, x\right) \circ \varphi_{\tau, x}^{ף}=\varphi_{t, x^{\prime}}^{\mathscr{V}}$.

Remark 5.3. The map $\varphi_{t, x}^{\mathscr{r}}$ is functorial: suppose we are given compatible maps $(G, X) \rightarrow\left(G^{\prime}, X^{\prime}\right)$ and $\mathscr{J} \rightarrow \mathscr{J}^{\prime}$; if a special point $x$ of $X$ is mapped to a special point $x^{\prime}$ of $X^{\prime}$, then we obtain a commutative diagram

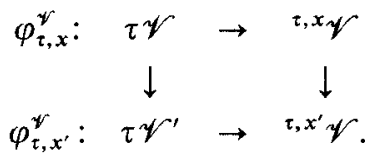

Remark 5.4. When $\mathscr{J}$ is a $G_{\mathbb{C}}^{\text {ad }}$-vector bundle on $\check{X}$ (rather than a $G_{\mathbb{C}^{-v e c t o r}}$ bundle), it is possible to give a more direct definition of ${ }^{\tau, x} \mathscr{J}$ (hence of ${ }^{\tau, x} \mathscr{V}$ ) and a characterization of $\varphi_{\tau, x}^{\mathscr{V}}$.

(a) The vector bundle $\mathscr{J}$ corresponds (by 4.3) to a representation $(r, V)$ of $P_{x}$, the subgroup of $G_{\mathbb{C}}^{\text {ad }}$ fixing $\beta(x)$. On applying $\tau$ we obtain a representation $\tau r$ of $\tau P_{x}$ on $V$. As in (2.8a), we can use ${ }^{\tau} \subseteq$ s to twist $\tau r: \tau P_{x} \rightarrow G L(V)$ and obtain a representation $\left.{ }^{\tau, x} r:{ }^{\tau, x} P_{x} \rightarrow G L{ }^{\tau, x} V\right)$ of ${ }^{\tau, x} P_{x}$. But (see 2.8a), ${ }^{\tau, x} P_{x}$ is the subgroup of ${ }^{\tau, x} G(\mathbb{C})$ fixing ${ }^{t} x$, and so ${ }^{\tau, x} r$ gives rise to a ${ }^{\tau, x} G_{\mathbb{C}^{-v e c t o r}}$ bundle $\mathscr{J}^{\prime}$ on $\check{X}$. Clearly $\mathscr{J}^{\prime}={ }^{\tau, x} \mathscr{J}$.

(b) Recall that $\mathscr{V}_{[x]}=\mathscr{J}_{x}$. The map $\varphi_{\tau, x}^{\mathscr{V}}$ is the unique isomorphism $\tau \mathscr{V} \rightarrow{ }^{\tau, x} \mathscr{V}$ lying over $\varphi_{t, x}$ and such that

(i) the action of $\varphi_{\tau, x}^{\mathscr{V}}$ on the fibre over $\tau[x]$ can be identified with the map $\tau(V \otimes \mathbb{C}) \rightarrow{ }^{\tau, x} V \otimes \mathbb{C}$ defined by $z_{\infty}(\tau)$;

(ii) $\varphi_{\tau, x}^{\mathscr{V}} \circ \tau(g)=(\tau, x) \circ \varphi_{t, x}^{\mathscr{V}}$ for all $g \in G(\mathbb{Q})_{+}^{-}$.

Moreover $\varphi_{\tau, x}^{\mathscr{V}}$ carries the natural filtration on $\tau \mathscr{V}$ (see 4.6) into the natural filtration on ${ }^{\tau, x} \mathscr{V}$.

We clarify the condition (i). The representation $r \circ \rho_{x}$ on $V$ defines a $C M$ motive $M$ over $\Phi$, and $H_{B}(M)=V$. The composite $c(\tau l M)^{-1} \circ(1 \otimes \tau) \circ c(l M)$ is a $\tau$-linear map $H_{B}(l M) \otimes \mathbb{C} \rightarrow H_{B}(\tau l M) \otimes \mathbb{C}$ (see $1.2 \mathrm{~b}$ ), and so it defines a linear map $\tau\left(H_{B}(M) \otimes \mathbb{C}\right) \rightarrow H_{B}(\tau t M) \otimes \mathbb{C}$, which we know is $z_{\infty}(\tau)$. But $\tau\left(H_{B}(M) \otimes \mathbb{C}\right)=\tau(V \otimes \mathbb{C})$ and (see 1.8) $H_{B}(\tau \imath M)={ }^{\tau, x} V$, and so $z_{\infty}(\tau)$ can be regarded as a map

$$
\tau\left(\mathscr{V}_{x}\right)=\tau(V \otimes \mathbb{C}) \rightarrow^{\tau, x} V \otimes \mathbb{C}={ }^{\tau, x} \mathscr{V}_{\tau_{x}}
$$

[It would be possible to state similar improvements of (5.2) for all automorphic vector bundles if we had a satisfactory theory of fractional CMmotives over $\mathbb{Q}$.]

Remark 5.4. For some automorphic vector bundles $\mathscr{V}(\mathscr{J})$ it is possible to prove (5.2) without using (3.10). For example, let $\mathscr{J}$ be $\mathscr{T}_{\dot{X}}$, the tangent bundle to $\check{X}$; for any $o \in \check{X}, \mathscr{J}$ corresponds to the adjoint representation of $P_{0}$ on $\operatorname{Lie}\left(P_{0}\right)$. Then $\mathscr{V}(\mathscr{J})$ is the tangent bundle to $S^{0}(G, X)$, and ${ }^{\tau, x} \mathscr{J}$ is the tangent bundle to ${ }^{\tau, x} \breve{X}$. Therefore the isomorphism of pro-varieties $\varphi_{\tau, x}: \tau S^{0}(G, X) \rightarrow S^{0}\left({ }^{\tau, x} G,{ }^{\tau, x} X\right)$ defines an equivariant isomorphism $\mathscr{T}\left(\varphi_{\tau, x}\right): \tau \mathscr{V}(\mathscr{T}) \stackrel{\approx}{\longrightarrow}(\tau, x)$. To show 
that this is the same as the map in (5.2), it suffices to show that it satisfies conditions (i) and (ii) of (5.4). Condition (ii) is obvious, and (i) can be shown by unwinding the various definitions, because $\mathscr{T}_{\bar{X}}$ is the $G$-vector bundle associated with the adjoint representation of $P_{0}$ on $\operatorname{Lie}\left(G_{\mathbb{C}}\right) / \operatorname{Lie}\left(P_{0}\right)$.

A similar remark applies if $\mathscr{J}$ is the bundle of $n$-jets of $\mathscr{T}_{\dot{X}}$.

Remark 5.6. When $\mathscr{J}$ arises from a representation $r: G_{\mathbb{C}} \rightarrow G L(V)$ of $G_{\mathbb{C}}$ (rather than of $P_{0}$ ), then $\varphi_{\tau, x}^{\mathscr{V}}$ carries the flat connection $\tau \nabla$ on $\tau \mathscr{V}$ (see 4.7) into the natural flat connection ${ }^{\tau, x} \nabla$ on ${ }^{\tau, x} \mathscr{V}$. It therefore defines an isomorphism of the local system $\tau V$ on $\tau S^{0}(G, X)$ (defined by the pair $(\tau \mathscr{V}, \tau V)$ ) with the local system ${ }^{\tau, x} \mathbf{V}$ on $S^{0}\left({ }^{\tau, x} G,{ }^{\tau, x} X\right)$ (defined by the pair $\left({ }^{\tau, x} \mathscr{W},{ }^{\tau, x} \nabla\right)$ ).

When $\mathscr{J}$ is defined by a real representation $(r, V)$ of $G_{\mathbf{R}}, \varphi_{\tau, x}$ defines an isomorphism of variations of real polarizable Hodge structures.

Finally, when $\mathscr{J}$ is defined by a rational representation $(r, V)$ of $G, \varphi_{t, x}^{\mathscr{V}}$ defines an isomorphism of variations of rational Hodge structures; moreover, it defines for each prime $l$ an isomorphism of $l$-adic sheaves.

Remark 5.7. Theorem 5.2 has a more down-to-earth interpretation. Let $\mathscr{V}=\mathscr{V}(\mathscr{J})$ be an automorphic vector bundle on $S^{0}(G, X)$, and let $f$ be an automorphic form on $X$ of type $\mathscr{J}$ and level $\Gamma$ with $\Gamma \in \tilde{\Sigma}(G)$. We can regard $f$ as an algebraic section of $\mathscr{V}_{\Gamma}$ over $S_{\Gamma}^{0}(G, X)$. Define the congruence subgroup ${ }^{\tau, x} \Gamma$ of ${ }^{\tau, x} G(\mathbb{Q})$ as in (1.7). Let $\tau$ be an automorphism of $\mathbb{C}$, and let $x$ be a special point of $X$. Then the theorem associates with $f$ an automorphic form $\tau, x f^{d f}=\tau f \circ\left(\varphi_{\tau, x}^{\mathscr{V}}\right)^{-1}$ on the Hermitian symmetric domain ${ }^{\tau, x} X$ of type ${ }^{\tau, x} \mathscr{J}$ and level ${ }^{\tau, x} \Gamma$; moreover, ${ }^{\tau, x}(f \circ(g))={ }^{\tau, x} f \circ\left({ }^{\tau, x} g\right)$ for all $g \in G(\mathbb{Q})_{+}^{-}$, and ${ }^{\tau, x} f\left(\left[{ }^{\tau} x\right]\right)$ can be related to $\tau(f([x]))$ (by means of $\left.z_{\infty}(\tau)\right)$ when $\mathscr{J}$ is a $G_{\mathbb{C}}^{\text {ad }}$-vector bundle.

\section{Proof of Theorem 3.10 for the symplectic group}

Let $V$ be a vector space over $\mathbb{Q}$, and let $\psi_{0}$ be a nondegenerate skew-symmetric form on $V$. The group $G=\operatorname{Sp}\left(V, \psi_{0}\right)$ of automorphisms of $V$ preserving $\psi_{0}$ is semisimple, and there is a unique conjugacy class $S^{+}$of homomorphisms $\mathbf{S} \rightarrow G^{\text {ad }}(\mathbb{R})^{+}$such that

(a) $\left(G, S^{+}\right)$is a pair defining a Shimura variety;

(b) each $h$ in $S^{+}$is of weight -1 ;

(c) the symmetric form $\psi_{0}\left(v, h(i) v^{\prime}\right)$ is positive definite for all $h \in S^{+}$.

Our goal in this section is to prove (3.10) for the pair $\left(G, S^{+}\right)$, but first we shall prove (5.2) for the automorphic vector bundle defined by the representation of $G$ on $V$.

Let $G_{1}$ be the group of symplectic similitudes $G \operatorname{Sp}\left(V, \psi_{0}\right)$ of $\left(V, \psi_{0}\right)$, and let $S^{ \pm}$be the Siegel double space in the sense of Deligne (1979), 1.3.1. Then $\left(G_{1}, S^{ \pm}\right)$is a pair defining a Shimura variety, and $\left(G, S^{+}\right)=\left(G_{1}, S^{ \pm}\right)^{+}$.

For an abelian variety $A$, we set $T A=\lim A_{m}$ (limit over all positive integers $m$ ordered by division), and we set $V^{f} A=T A \otimes \mathbb{Q}$. Note that $V^{f} A$ depends only on the isogeny class of $A$. Consider triples $(A, \psi, k)$ consisting of an abelian 
variety $A$ over $\mathbb{C}$ (defined up to isogeny), a polarization $\psi$ of $A$, and an isomorphism $k: V^{f}(A) \stackrel{\approx}{\longrightarrow} V\left(\mathbb{A}^{f}\right)$ carrying $\psi$ to $\psi_{0}$. We define $\mathfrak{U}\left(V, \psi_{0}\right)$ to be the set of isomorphism classes of triples of this form for which there is an isomorphism of symplectic spaces $\left(H_{1}(A, \mathbb{Q}), \psi\right) \stackrel{\approx}{\longrightarrow}\left(V, \psi_{0}\right)$. The group $G_{1}\left(\mathbb{A}^{f}\right)$ acts on $\mathfrak{U}\left(V, \psi_{0}\right)$ according to the rule:

$$
[A, \psi, k] g=\left[A, \psi, g^{-1} k\right], \quad g \in G_{1}\left(\mathbb{A}^{f}\right) .
$$

Lemma 6.1. There is a bijection $S\left(G_{1}, S^{ \pm}\right)(\mathbb{C}) \rightleftharpoons \mathfrak{\approx} \mathfrak{\longrightarrow}\left(V, \psi_{0}\right)$ commuting with the actions of $G\left(\mathbb{A}^{f}\right)$.

Proof. Corresponding to

$$
[x, g] \in S\left(G_{1}, S^{ \pm}\right)(\mathbb{C})=G_{1}(\mathbb{Q}) \backslash X \times G\left(\mathbb{A}^{f}\right)
$$

we choose $A$ to be the abelian variety (defined up to isogeny) associated with the rational Hodge structure $\left(V, h_{x}\right)$. Then $\psi_{0}$ defines a polarization $\psi$ of $A$, and we define $k$ to be the composite $V^{f}(A) \stackrel{=}{\longrightarrow} V \otimes \mathbb{A}^{f} \stackrel{\mathrm{g}^{-1}}{\longrightarrow} V\left(\mathbb{A}^{f}\right)$. (See for example Milne and Shih (1982b), 2.3.)

Let $x$ be a special point of $S^{+}$. Then $h_{x}: \mathbb{S} \rightarrow G_{1 \mathbb{R}}$ defines a map $\rho_{x}: \Xi \rightarrow G_{1}$ $=G \operatorname{Sp}\left(V, \psi_{0}\right)$, and we can use $\rho_{x}$ and ${ }^{\tau} \subseteq$ to twist $\left(V, \psi_{0}\right)$. In this way we obtain a new pair $\left({ }^{\tau, x} V,{ }^{\tau, x} \psi_{0}\right)$ with $G \operatorname{Sp}\left({ }^{\tau, x} V,{ }^{\tau, x} \psi_{0}\right)={ }^{\tau, x} G_{1}$. Let $(A, \Psi, k)$ be the triple associated with $[x, g]$. Then $H_{1}(\tau A, \mathbb{Q})={ }^{\tau, x} V$ and $\tau \psi={ }^{\tau, x} \psi_{0}$ (cf. 1.8). The map $\varphi_{\tau, x}: \tau S^{0}(G, X) \rightarrow S^{0}\left({ }^{\tau, x} G,{ }^{\tau, x} X\right)$ corresponds under the bijective in (6.1) to $\tau[A, \psi, k] \mapsto\left[\tau A, \tau \psi,{ }^{\mathrm{t}} k\right]$ where ${ }^{\mathrm{t}} k$ is the composite of the maps

$$
V^{f}(\tau A) \stackrel{\tau^{-1}}{\longrightarrow} V^{f}(A) \stackrel{k}{\longrightarrow} V\left(\mathbb{A}^{f}\right) \stackrel{\operatorname{sp}(\tau)}{\longrightarrow}, x \in\left(\mathbb{A}^{f}\right) .
$$

See [ibid., 7.16].

Just as in (4.7), the pair $\left(V, \psi_{0}\right)$ defines a polarized variation of rational Hodge structures $\left(\mathbf{V}, \Psi_{0}\right)$ on $S\left(G_{1}, S^{ \pm}\right)$. We use ${ }^{\vee}$ to denote a linear dual.

Proposition 6.2. There is a canonical abelian scheme $\pi: \mathscr{A} \rightarrow S\left(G_{1}, S^{ \pm}\right)$over $S\left(G_{1}, S^{ \pm}\right)$and a polarization $\Psi$ of $\mathscr{A}$ such that $\left(\left(R^{1} \pi_{*} \mathbb{Q}\right)^{\vee}, \Psi\right)=\left(\mathbf{V}, \Psi_{0}\right)$.

Proof. Choose a lattice $V_{0} \subset V$ such that $\psi_{0}$ is integral on $V_{0}$, and for any integer $N \geqq 3$, define $K(N)$ to be the subgroup of $G\left(\mathbb{A}^{f}\right)$ stabilizing $V_{0} \otimes \mathbb{A}^{f}$ and acting as the identity map on $V_{0} / N V_{0}$. Then $S_{K(N)}\left(G_{1}, S^{ \pm}\right)$is the moduli scheme over $\mathbb{C}$ for polarized abelian varieties of dimension $\operatorname{dim}(V) / 2$ and degree the discriminant of $\psi_{0}$ on $V_{0}$, and we can take $\mathscr{A}_{K(N)}$ to be the universal abelian scheme over $S_{K(N)}\left(G_{1}, S^{ \pm}\right)$(Mumford (1965), 7.9). The subgroups $K(N)$ are cofinal among compact-open subgroups of $G_{1}\left(\mathbb{A}^{f}\right)$, and we define $\mathscr{A}$ to be the inverse limit, $\lim _{\leftarrow} \mathscr{A}_{K(N)}$.

Remark 6.3. (a) For each point $s$ of $S\left(G_{1}, S^{ \pm}\right)$, the fibre $\mathscr{A}_{S}$ is the abelian variety attached to $s$ in (6.1). 
(b) From $\mathscr{A}$ we get an isomorphism $k_{l}:\left(R^{1} \pi_{*} \mathbb{Q}_{l}\right)^{\vee} \stackrel{\approx}{\longrightarrow} \mathbf{V}\left(\mathbb{Q}_{l}\right)$ for each $l$, where $\mathbf{V}$ is the rational local system on $S\left(G_{1}, S^{ \pm}\right)$defined by $V$. Write $V_{l}(\mathscr{A})$ for $\left(R^{1} \pi_{*} \mathbb{Q}_{l}\right)^{\vee}$; then we can think of the family $k=\left(k_{l}\right), k_{l}: V_{l}(\mathscr{A}) \rightarrow \mathrm{V}\left(\mathbb{Q}_{l}\right)$, as being a level structure on $\mathscr{A}$. The triple $(\mathscr{A}, \Psi, k)$ has the following universal property: for any $G_{1}\left(\mathbb{A}^{f}\right)$-scheme $S$ over $\mathbb{C}$ and triple $\left(\mathscr{A}^{\prime}, \Psi^{\prime}, k^{\prime}\right)$ over $S$ such that $\left(\mathscr{A}^{\prime}, \Psi^{\prime}, k^{\prime}\right)_{s} \in \mathfrak{A}\left(V, \Psi_{0}\right)$ for all $s \in S(\mathbb{C})$, there exists a unique isomorphism $\alpha: S \rightarrow S\left(G_{1}, S^{ \pm}\right)$of $G\left(\mathbb{A}^{f}\right)$-schemes such that $\alpha^{*}(\mathscr{A}, \Psi, k)=\left(\mathscr{A}^{\prime}, \Psi^{\prime}, k^{\prime}\right)$.

This can be proved easily using the universal property of each $\mathscr{A}_{K(N)}$.

When we apply the construction in (6.2) and $(6.3 \mathrm{~b})$ to $\left({ }^{\tau, x} V,{ }^{\tau, x} \psi\right)$, we get a polarized abelian scheme $\left({ }^{\tau, x} \mathscr{A},{ }^{\tau, x} \Psi\right)$ over $S\left({ }^{\tau, x} G_{1}, S^{ \pm}\right)$with a level structure ${ }^{\tau, x} k$, where ${ }^{\tau, x} k_{l}$ is an isomorphism $V_{l}(\mathscr{A}) \stackrel{\approx}{\longrightarrow}{ }^{t, x} \mathbf{V}(\mathbb{Q})$.

Proposition 6.4. There is a unique isomorphism $\varphi_{\tau, x}^{\mathscr{A}}: \tau \mathscr{A} \rightarrow^{\tau, x} \mathscr{A}$ such that

(a) the following diagram commutes

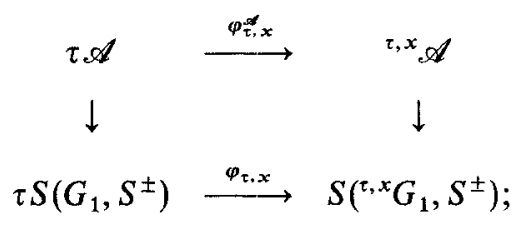

(b) $\varphi_{t, x}^{\infty}$ sends $\tau \Psi$ to ${ }^{\tau, x} \Psi$

(c) the following diagram commutes

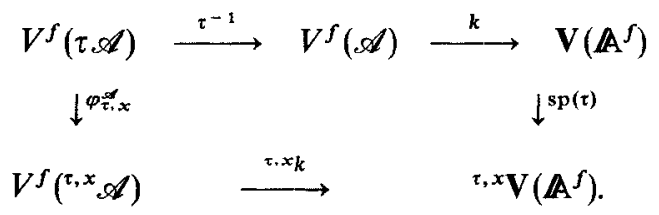

Proof. Apply the universal property of ${ }^{\tau, x} \mathscr{A}$ (and use that $\left.V \otimes \mathbb{A}^{f}\right)=^{\tau, x} V \otimes \mathbb{A}^{f}$ ).

Corollary 6.5. There is a unique isomorphism $\varphi_{\tau, x}^{\vee}: \tau \mathscr{V} \stackrel{\approx}{\longrightarrow, x} \mathscr{V}$ lying over $\varphi_{\tau, x}$ and such that

(i) the restriction of $\varphi_{\tau, x}^{\vee}$ to the fibre over $[x, 1]$ can be identified with the map $\tau V(\mathbb{C}) \rightarrow^{\tau, x} V(\mathbb{C})$ defined by $z_{\infty}(\tau)$;

(ii) $\varphi_{\tau, x}^{\mathfrak{V}} \circ \tau(g)=\left(^{\tau, x} g\right) \circ \varphi_{\tau, x}^{ף}$ for all $g \in G_{1}\left(\mathbb{A}^{f}\right)$.

Proof. The uniqueness is obvious. For the existence, note that $\mathscr{V}=\mathscr{H}_{1}^{d R}(\tau \mathscr{A})$ and $^{\tau, x} \mathscr{V}=\mathscr{H}_{1}^{d R}(\tau, x \mathscr{A})$, and so $\varphi_{\tau, x}^{\mathscr{v}}$ can be taken to be $\mathscr{H}_{1}^{d R}\left(\varphi_{\tau, x}^{\mathscr{A}}\right)$.

Corollary 6.6. Theorem 5.2 is true for the automorphic vector bundle $\mathscr{V}$ defined by the representation of $G$ on $V$.

Proof. The restriction of $\varphi_{\tau, x}^{\mathscr{V}}$ to $\tau S^{0}(G, X)$ has the correct properties. For a second special point $x^{\prime}$, it is not difficult to trace through the various constructions and see that $\varphi^{\mathscr{V}}\left(\tau ; x^{\prime}, x\right) \circ \varphi_{\tau, x}^{\vee}=\varphi_{\tau, x^{\prime}}^{\vee}$.

Corollary 6.7. Theorem 3.10 is true for $\left(G, S^{+}\right)$. 
Proof. This follows from (6.6) because (3.4) allows us to identify $Y^{0}\left(G, S^{+}\right)$and $\left.Y^{0}{ }^{\tau}, x \in,{ }^{\tau, x} S^{+}\right)$respectively with the spaces of trivializations of $\mathscr{V}$ and ${ }^{\tau, x} \mathscr{V}$ preserving the polarizations.

\section{Proof of Theorem 3.10 for connected Shimura varieties of abelian type}

Lemma 7.1. Let $f:(G, X) \rightarrow\left(G^{\prime}, X^{\prime}\right)$ be an embedding of pairs defining connected Shimura varieties. If Theorem 3.10 is true for $\left(G^{\prime}, X^{\prime}\right)$, then it is also true for $(G, X)$.

Proof. Let $Y$ be the inverse image of $\tau Y^{0}\left({ }^{\tau, x} G,{ }^{\tau, x} X\right)$ under $\varphi_{\tau, x}^{Y^{\prime}}$,

$$
\begin{array}{ccc}
\tau Y^{0}(G, X) & \hookrightarrow & Y^{0}\left(G^{\prime}, X^{\prime}\right) \\
& & \downarrow^{\varphi_{t, x}^{Y^{\prime}}} \\
\tau Y^{0}\left(^{\tau, x} G,{ }^{\mathfrak{r}, x} X\right) & \hookrightarrow & Y^{0}\left({ }^{\mathfrak{r}, x} G^{\prime,},{ }^{\tau, x} X^{\prime}\right) .
\end{array}
$$

Then $Y$ is a closed subset containing $\tau y$, and it contains a fibre of the map $\pi: \tau Y^{0}(G, X) \rightarrow \tau S^{0}(G, X)$ whenever it contains a single point; moreover, its image in $\tau S^{0}(G, X)$ is stable under the action of $\mathscr{G}(G)$. The same argument as in the proof of the uniqueness statement in (3.10) now shows that $Y=\tau Y^{0}(G, X)$. Therefore the restriction of $\varphi_{\tau, x}^{Y}$ to $\tau Y^{0}(G, X)$ is an isomorphism satisfying the conditions (i) (iii) of the theorem.

If $x^{\prime}$ is a second special point of $X$, then the restriction of $\varphi^{Y^{\prime}}\left(\tau ; x^{\prime}, x\right)$ to $Y^{0}\left({ }^{\tau, x} G,{ }^{\tau, x} X\right)$ is $\varphi^{Y}\left(\tau ; x^{\prime}, x\right)$, and so the equality $\varphi^{Y}\left(\tau ; x^{\prime}, x\right) \circ \varphi_{\tau, x}^{Y}=\varphi_{\tau, x^{\prime}}^{Y}$ is implied by the similar equality for $Y\left(G^{\prime}, X^{\prime}\right)$.

Recall (Milne and Shih (1982b), $\S 1$, especially 1.3$)$ that if $(G, X)$ is primitive of abelian type, then there is an embedding $(G, X) \hookrightarrow\left(\operatorname{Sp}(V, \psi), S^{+}\right)$for some symplectic space $(V, \psi)$. Therefore (6.7) and the lemma and show that Theorem 3.10 holds for every pair $(G, X)$ that is primitive of abelian type. By definition, for every pair $(G, X)$ of abelian type, there exists a family of pairs $\left(G_{i}, X_{i}\right)$, primitive of abelian type, and a morphism $\left(\Pi G_{i}, \Pi X_{i}\right) \rightarrow(G, X)$ with $\Pi G_{i} \rightarrow G$ an isogeny [ibid. p. 293]. Therefore, the next two lemmas complete the proof of Theorem 3.10 for pairs $(G, X)$ of abelian type.

Lemma 7.2. Let $\left(G^{\prime}, X^{\prime}\right) \rightarrow(G, X)$ be a morphism such that $G^{\prime} \rightarrow G$ is an isogeny. If Theorem 3.10 is true for $\left(G^{\prime}, X^{\prime}\right)$, then it is also true for $(G, X)$.

Proof. Note that in this case $G^{\prime \text { ad }}=G^{\text {ad }}$ and $X=X^{\prime}$. Moreover, $S^{0}(G, X)$ is the quotient of $S^{0}\left(G^{\prime}, X\right)$ by the kernel $\mathscr{G}\left(G^{\prime}, G\right)$ of the map $G^{\prime a d}(\mathbb{Q})^{+\wedge} \rightarrow G^{\text {ad }}(\mathbb{Q})^{+\wedge}$. Similarly, $Y^{0}(G, X)$ is the quotient of $Y^{0}\left(G^{\prime}, X\right)$ by the kernel of the surjective homomorphism $\mathscr{G}\left(G^{\prime}\right) \rightarrow \mathscr{G}(G)$. The map $\varphi_{\tau, x}^{Y}$ for $(G, X)$ can therefore be obtained from the corresponding map $\varphi_{\tau, x^{\prime}}^{Y^{\prime}}$ for $\left(G^{\prime}, X^{\prime}\right)$ by passing to the quotient.

Lemma 7.3. For $i=1, \ldots, n$, let $\left(G_{i}, X_{i}\right)$ be a pair defining a connected Shimura variety, and let $G=\Pi G_{i}$ and $X=\Pi X_{i}$. If Theorem 3.10 is true for each pair $\left(G_{i}, X_{i}\right)$ then it is true for $(G, X)$.

Proof. This is obvious. 
Proposition 7.4. Theorem 3.10 is true for all connected Shimura varieties of abelian type.

Proof. We have already noted that this follows from (6.7) and the preceding lemmas.

In fact, in the proof of the general case of (3.10) we shall need to use (7.4) only for connected Shimura varieties associated with groups $G$ of type $A_{1}$ (then $\tilde{G}=S L_{1}(B)$ for $B$ a quaternion algebra defined over a totally real field $F$ ).

We now make some remarks that will assist us in the next two sections in the proof of the general case.

Lemmas 7.2 and 7.3 show that it suffices to prove Theorem 3.10 for $(G, X)$ with $G$ simply connected and $G^{\text {ad }} \mathbb{Q}$-simple.

For the remainder of this section, we assume that $G$ is simply connected. Let $x$ be a special point of $X$. For each homomorphism $r: G(\mathbb{Q}) \rightarrow G(\mathbb{C})$, we define the principal $G_{\mathbb{C}^{-}}$bundle $Y(r)$ on $S^{0}(G, X)$ to be

$$
Y(r)=G(\mathbb{Q}) \backslash X \times G(\mathbb{C}) \times G\left(\mathbb{A}^{f}\right)
$$

where $q \in G(\mathbb{Q})$ acts on $(x, c, a) \in X \times G(\mathbb{C}) \times G\left(\mathbb{A}^{f}\right)$ according to the rule

$$
q(x, c, a)=(q x, r(q) \cdot c, q a) .
$$

There is an obvious flat connection $\nabla(r)$ on $Y(r)$, and an action $a(r)$ of $G\left(\mathbb{A}^{f}\right)$ on $(Y(r), \nabla(r))$. Let $y(r)$ be the point $[x, 1,1]$ on $Y(r)$.

Our next lemma was suggested by a similar result in Harris (1986), 3.6.

Lemma 7.5. The map $r \mapsto(Y(r), \nabla(r), a(r), y(r))$ gives a bijection between the set of homomorphisms $r: G(\mathbb{Q}) \rightarrow G(\mathbb{C})$ and the set of isomorphism classes of quadruples $(Y, \nabla, a, y)$ consisting of a principal $G_{\mathbb{C}}$-bundle $Y$, a flat connection $\nabla$ on $Y$, an action $a$ of $G\left(\mathbb{A}^{f}\right)$ on $(Y, \nabla)$, and a point $y$ lying over $[x]$.

Proof. Suppose we are given a quadruple $(Y, \nabla, a, y)$. Because $X$ is simply connected, there is a map $\psi: X \times G(\mathbb{C}) \times G\left(\mathbb{A}^{f}\right) \rightarrow Y$ compatible with the projections to $S^{0}(G, X)(\mathbb{C})$, the flat structures, and the actions of $G\left(\mathbb{A}^{f}\right)$ and $G(\mathbb{C})$. When we normalize $\psi$ by requiring that $\psi(x, 1,1)=y$, then it is uniquely determined. The map $r$ corresponding to $(Y, \nabla, a, y)$ is determined by the rule:

$$
\psi(q x, 1, q)=r(q)^{-1} y, \quad \text { all } q \in G(\mathbb{Q}) .
$$

Remark 7.6. Let $x$ be a special point of $X$. Corresponding to the pair $\left({ }^{\tau, x} G,{ }^{\tau, x} X\right)$ and the special point ${ }^{\tau} x$ of ${ }^{r, x} X$, we have a quadruple $\left(Y^{0}\left({ }^{\tau, x} G,{ }^{\tau, x} X\right),{ }^{\tau, x} \nabla,{ }^{\tau, x} a,{ }^{\tau} y\right)$. On pulling back by $\varphi_{\tau, x}^{0}$, applying $\tau^{-1}$, and finally pulling back again relative to the map $g \mapsto^{\tau, x} g: G(\mathbb{C}) \rightarrow^{\tau, x} G(\mathbb{C})$, we obtain a similar quadruple on $S^{0}(G, X)$, which we denote by $\left({ }^{x} Y,{ }^{x} \nabla,{ }^{x} a,{ }^{x} y\right)$. According to (7.5), this corresponds to a homomorphism $r_{x}: G(\mathbb{Q}) \rightarrow G(\mathbb{C})$, and to prove the existence of an isomorphism $\varphi_{\tau, x}^{Y}$ satisfying (i)-(iii) of Theorem 3.10 it suffices to show that this homomorphism is the natural inclusion. 


\section{First completion of the proof of Theorem 3.10}

Let $(G, X)$ be a pair defining a connected Shimura variety, and assume that $G$ is simply connected and that $G^{\text {ad }}$ is $Q$-simple. Then $G=\operatorname{Res}_{F / \mathbb{Q}} H$ with $H$ an absolutely almost simple group over a totally real number field $F$. Let $x$ be a special point of $X$, and let $T$ be a maximal torus of $G$ such that $h_{x}$ factors through $(T / Z)(\mathbb{R})$. There is a maximal torus $T^{\prime}$ of $H$ such that $T=\operatorname{Res}_{F / \mathbb{Q}} T^{\prime}$.

For any totally real number field $F^{\prime}$ containing $F$ and such that $T_{F^{\prime}}^{\prime}$ splits over a quadratic imaginary extension $L$ of $F^{\prime}$, we can write

$$
\operatorname{Lie}\left(H_{L}\right)=\operatorname{Lie}\left(T_{L}^{\prime}\right) \otimes\left(\bigotimes_{\alpha \in R} \operatorname{Lie}\left(H_{L}\right)_{\alpha}\right)
$$

where $R=R\left(H_{\mathbb{C}}, T_{\mathscr{C}}^{\prime}\right)$. Recall that a root $\alpha$ is said to be totally compact if it is a compact root of $\left(H_{F^{\prime}} \otimes_{F^{\prime}, \sigma} \mathbb{R}\right)$ for all embeddings $\sigma: F^{\prime} \hookrightarrow \mathbb{R}$. For each root $\alpha$ that is not totally compact, the subgroup $H_{\alpha}$ is defined to be the connected subgroup of $H_{F}$, such that

$$
\operatorname{Lie}\left(H_{\alpha}\right)_{L}=\operatorname{Lie}\left(T_{L}^{\prime}\right) \oplus \operatorname{Lie}\left(H_{L}\right)_{\alpha} \oplus \operatorname{Lie}\left(H_{L}\right)_{-\alpha} .
$$

Proposition 8.1. If $F^{\prime}$ is chosen to be sufficiently large, then $H(F)$ is contained in the subgroup of $H(\Phi)$ generated by $\bigcup H_{\alpha}\left(F^{\prime}\right)$, where the union is taken over all nontotally compact roots $\alpha$.

Proof. This is stated (without proof) in Borovoi (1983/84), 3.21.

Choose a field $F^{\prime}$ as in the proposition, and let $G_{*}=\operatorname{Res}_{F^{\prime} / \mathrm{Q}} H$ and, for each nontotally compact root $\alpha$ of $G$, let $G_{\alpha}=\operatorname{Res}_{F^{\prime} / Q} H_{\alpha}$. There are natural maps $(G, X) \hookrightarrow\left(G_{*}, X_{*}\right)$ and $\left(G_{\alpha}, X_{\alpha}\right) \hookrightarrow\left(G_{*}, X_{*}\right)$, each $\alpha$. Write $x_{*}$ for the image of $x$ in $X_{*}$, and write $x_{\alpha}$ for it regarded as an element of $X_{\alpha}$. Then the construction in (7.6) applied to the triples $\left(G_{*}, X_{*}, x_{*}\right)$ and $\left(G_{\alpha}, X_{\alpha}, x_{\alpha}\right)$ gives us homomorphisms $r_{x *}: G_{*}(\mathbb{Q}) \rightarrow G_{*}(\mathbb{C})$ and $r_{x_{\alpha}}=G_{\alpha}(\mathbb{Q}) \rightarrow G_{\alpha}(\mathbb{C})$. Clearly the maps $r_{x}$, $r_{x_{*}}$, and $r_{x_{x}}$ are compatible with the inclusions $G \hookrightarrow G_{*}$ and $G_{\alpha} \hookrightarrow G_{*}$; that is, the following diagram commutes,

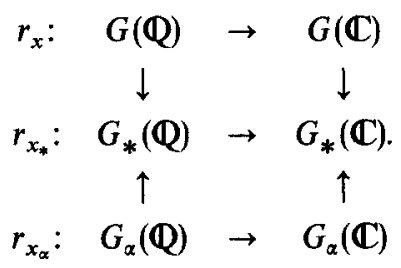

Since we know Theorem 3.10 for groups of type $A_{1}$, each map $r_{x_{x}}$ is the natural inclusion. The proposition shows that $G(\mathbb{Q}) \subset \cup G_{\alpha}(\mathbb{Q})$, and so $r_{x}$ must also be the natural inclusion.

To complete the proof of (3.10), we have to show that if $x^{\prime}$ is a second special point, then $\varphi^{Y}\left(\tau ; x^{\prime}, x\right) \circ \varphi_{\tau, x}^{Y}=\varphi_{\tau, x^{\prime}}^{Y}$. Each is a map $\tau Y^{0}(G, X) \rightarrow Y^{0}\left({ }^{\tau}, x^{\prime} G, \tau, x^{\prime} X\right)$ compatible with the homomorphism $g \mapsto^{\tau, x} g: G\left(\mathbb{A}^{\prime}\right) \rightarrow^{\tau, x} G\left(\mathbb{A}^{\prime}\right)$. To prove that the maps are equal, it suffices to show that they agree on a single point (compare 
the proof of the uniqueness assertion of (3.10)). If $x$ and $x^{\prime}$ both lie in $X_{\alpha}$ for some noncompact root $\alpha$ as above, then the maps agree on the whole of $Y^{0}\left(G_{\alpha}\right.$, $\left.X_{\alpha}\right)$, and so they are equal. If $x^{\prime}=g x$ for some $g \in G(Q)$, then one can show directly that the two maps agree on the $\tau y$, where $y$ is the image of $x$ in $Y^{0}(G$, $X)$. To complete the proof, we need to recall one final result.

Proposition 8.2. Let $x$ and $x^{\prime}$ be special points of $X$. If $F^{\prime}$ is chosen large enough, then there exists a sequence $(x=) x_{0}, \ldots, x_{m}\left(=x^{\prime}\right)$ of special points of $X_{*}$ and an element $g \in G_{*}(\mathbb{Q})$ such that

(a) for each positive $i<m$, there is a noncompact root $\alpha$ such that $x_{i-1}$ and $x_{i}$ lie in $X_{\alpha}$;

(b) $g x_{m-1}=x_{m}$.

Proof. See Borovoi (1983/84), 1.18 or Milne (1983), pp. 260-261.

We now complete the proof for a general pair of special points $\left(x, x^{\prime}\right)$. After replacing $(G, X)$ with a suitable pair $\left(G_{*}, X_{*}\right)$, we can assume that there exist points $x_{0}, \ldots, x_{m}$ and an element $q$ as in the statement of the proposition. The remarks preceding the proposition show that

$$
\varphi^{Y}\left(\tau ; x_{i}, x_{i-1}\right) \circ \varphi_{\tau, x_{i-1}}^{Y}=\varphi_{t, x_{i}}^{Y} \quad \text { for } i=1, \ldots, m .
$$

On multiplying these equalities, we find that

$$
\varphi_{\tau, x^{\prime}}^{Y}=\varphi^{Y}\left(\tau ; x_{m}, x_{m-1}\right) \circ \ldots \circ \varphi^{Y}\left(\tau ; x_{1}, x_{0}\right) \circ \varphi_{\tau, x}^{Y} .
$$

It is clear from the definition of the maps $\varphi^{Y}(\tau ; \ldots)$ (see 3.7) that

$$
\varphi^{Y}\left(\tau ; x_{m}, x_{m-1}\right) \circ \ldots \circ \varphi^{Y}\left(\tau ; x_{1}, x_{0}\right)=\varphi^{Y}\left(\tau ; x_{m}, x_{0}\right)
$$

and so this completes the proof of Theorem 3.10 (assuming 8.1).

\section{Second completion of the proof of Theorem 3.10}

Again we assume that $(G, X)$ is a pair defining a connected Shimura variety with $G$ simply connected and $G^{\text {ad }} \mathbb{Q}$-simple. Let $x$ be a special point $X$, and let $r_{x}: G(\mathbb{Q}) \rightarrow G(\mathbb{C})$ be the map defined in (7.6). If we can show that $r_{x}$ is the natural inclusion map, then the same argument as in the previous section will complete the proof of Theorem 3.10 .

Now let $r: G^{\text {ad }} \rightarrow G L(V)$ be a representation of $G^{\text {ad }}$. We can twist this to obtain a representation ${ }^{\tau, x} r:{ }^{\tau, x} G^{\text {ad }} \rightarrow G L{ }^{\tau, x} V$ ). This defines the automorphic vector bundle ${ }^{r, x} \mathscr{V}$ on $\left.S^{0}{ }^{\tau, x} G,{ }^{\tau, x} X\right)$ together with its flat connection ${ }^{\tau, x} \nabla$ and action ${ }^{\tau, x} a$ of ${ }^{\tau, x} G\left(\mathbb{A}^{f}\right)$. On pulling this back by $\varphi_{\tau, x}^{0}$ to $\tau S^{0}(G, X)$ and applying $\tau^{-1}$, we obtain a vector bundle ${ }^{x} \mathscr{V}$ on $S^{0}(G, X)$ together with a flat connection ${ }^{x} \nabla$ and action ${ }^{x} a$ of $G\left(\mathbb{A}^{f}\right)$. From the triple $\left({ }^{x} \mathscr{V},{ }^{x} \nabla,{ }^{x} a\right)$ we obtain a representation $r_{x}(V)$ of $G(\mathbb{Q})$ on $V \otimes \mathbb{C}$. Note that Theorem 5.2 would imply that $r_{x}(V)$ is simply the map induced by $r$. 
Lemma 9.1. Let $r: G \rightarrow G L(V)$ be a representation of $G$ factoring through $G_{\mathbb{C}}^{\text {ad }}$. With the above notations, there is a commutative diagram

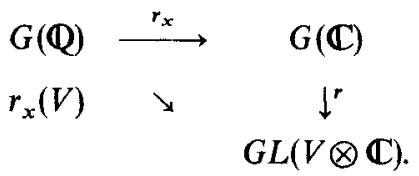

Proof. This simply says that the above construction of $r_{x}(V)$ is compatible with the construction in (7.6) of $r_{x}$.

Lemma 9.2. Consider the representation $r$ of $G^{\text {ad }}$ on $\mathfrak{g} \stackrel{d f}{=} \operatorname{Lie}(G)$. If for all triples $(G, X, x), r_{x}(\mathfrak{g})$ is the restriction of $r$ to $G^{\text {ad }}(\mathbb{Q})$, then $r_{x}: G(\mathbb{Q}) \rightarrow G(\mathbb{C})$ is the natural inclusion.

Proof. From the preceding lemma, we get a commutative diagram

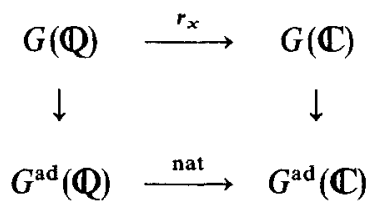

in which the map in the lower row is the natural inclusion. This implies that the map $\gamma: g \mapsto r_{x}(g) \cdot g^{-1}$ has image contained in the centre $Z(\mathbb{C})$ of $G(\mathbb{C})$. It is moreover a homomorphism. If we knew that $G(\mathbb{Q}) / Z(\mathbb{Q})$ was a simple group the proof would now be complete because we would have that $\gamma(G(\mathbb{Q}))=\{1\}$. Since we do not know it, we instead must argue as in Milne (1983), p. 251-252. Fix a $g \in G(\mathbb{Q})$. Because $G(\mathbb{Q})$ is generated by the groups $T_{x^{\prime}}(\mathbb{Q})$ with $x^{\prime}$ running through the special points of $X$ [ibid. 3.10], we can assume that $g$ lies in some $T_{x^{\prime}}(\mathbb{Q})$. If we write $G=\operatorname{Res}_{F / \mathbb{Q}} H$ and pass to a larger totally real field $F^{\prime}$, then we obtain inclusions $(G, X) \hookrightarrow\left(G_{*}, X_{*}\right)$ and $G_{\alpha} \hookrightarrow G_{*}$ with $G_{\alpha}$ a reductive group of type $A_{1}$ and $T_{x^{\prime}} \subset G_{\alpha}$. Moreover, after possibly enlarging the totally real field $F$ again, we can assume that $G_{\alpha} \otimes \mathbb{Q}_{l}$ is isotropic. On applying the above construction to the pair $\left(G_{*}, X_{*}\right)$ we obtain a map $\gamma_{*}: G_{*}(\mathbb{Q}) \rightarrow Z_{*}(\mathbb{C})$ whose restriction to $G(\mathbb{Q})$ is $\gamma$. According to Platonov and Rapincuk (1979), $G_{\alpha}(\mathbb{Q}) / Z_{\alpha}(\mathbb{Q})$ is simple, and so $\gamma_{*}$ is trivial on $G_{\alpha}(\mathbb{Q})$. As $g \in G_{\alpha}(\mathbb{Q})$, this shows that $\gamma(\mathrm{g})=1$.

It remains to verify that $r_{x}(\mathfrak{g}): G^{\text {ad }}(\mathbb{Q}) \rightarrow G L(\mathfrak{g})$ is given by the adjoint representation. Recall that $S^{0}(G, X)=G(\mathbb{Q}) \backslash X \times G\left(\mathbb{A}^{\mathcal{S}}\right)$, and write $\pi$ for the projection $X \times G\left(\mathbb{A}^{f}\right) \rightarrow S^{0}(G, X)$. For any $G\left(\mathbb{A}^{f}\right)$-local system $\mathbf{V}$ on $S^{0}(G, X), \pi^{*}(\mathbf{V})$ is spanned by its space of $G\left(\mathbb{A}^{f}\right)$-invariant global sections, and this last space can be identified with $\mathbf{V}_{x}$ for any $x \in X$. Therefore, from any such local system and point $x$, we obtain a representation $G(\mathbb{Q}) \rightarrow G L\left(\mathbf{V}_{x}\right)$. Because of the dictionary recalled in $(4.7)$, a $G\left(\mathbb{A}^{f}\right)$-vector bundle with flat connection $(\mathscr{K}, \nabla)$ also gives rise to a representation of $G(\mathbb{Q})$ on the fibre $\mathscr{V}_{x}$. 
Lemma 9.3. Let $(\mathscr{V}, \nabla)$ be a $G\left(\mathbb{A}^{f}\right)$-vector bundle with flat connection on $S^{0}(G, X)$, and assume that $\mathscr{V}$ is a $G\left(\mathbb{A}^{f}\right)$-subbundle of an automorphic vector bundle $\mathscr{V}(\mathscr{F})$ on $S^{0}(G, X)$. Then the representation $r: G(\mathbb{Q}) \rightarrow G L\left(\mathscr{V}_{x}\right)$ is continuous for the complex topology; if it is trivial on $Z(\mathbb{Q})$, then it is induced by a morphism of algebraic groups $G_{\mathbb{Q}}^{\text {ad }} \rightarrow G L\left(\mathscr{V}_{x}\right)$.

Proof. The first assertion follows from the fact that the representation $r$ of $G(\mathbb{Q})$ on $\mathscr{V}_{x}$ can be realized as a subrepresentation of the representation of $G(\mathbb{R})$ on $\Gamma\left(X, \beta^{*}(\mathscr{J})\right)$, which is obviously continuous. For the second, note that the homomorphism $r^{\text {ad }}: G(\mathbb{Q}) / Z(\mathbb{Q}) \rightarrow G L\left(\mathscr{V}_{x}\right)$ extends by continuity to a homomorphism $\hat{r}: G^{\text {ad }}(\mathbb{R})^{+} \rightarrow G L\left(\mathscr{V}_{x}\right)$. Let $r^{\prime}$ be the homomorphism of algebraic groups $G_{\mathbb{R}}^{\text {ad }} \rightarrow G L\left(\mathscr{V}_{x}\right)$ such that $\operatorname{Lie}\left(r^{\prime}\right)=\operatorname{Lie}(\hat{f})$. Then $r^{\prime}(\mathbb{R})$ and $\hat{r}$ agree on an open subgroup of $G^{\text {ad }}(\mathbb{R})^{+}$, and so they agree on the whole of $G^{\text {ad }}(\mathbb{R})^{+}$.

For a vector bundle $\mathscr{V}$ on a space, write $\mathscr{J} e t^{n}(\mathscr{V})$ for the bundle of jets of length $n$ of $\mathscr{V}$ (see Grothendieck (1967), IV.16.8).

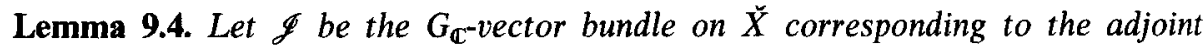
representation of $G$ on $\operatorname{Lie}(G)$. Then there is an equivariant embedding $\mathscr{J} \hookrightarrow \mathscr{L e t}^{2}\left(T_{\tilde{x}}\right)$.

Proof. See Harris (1985), p. 172.

We now complete the proof of the theorem. Let $x$ be a special point of $X$. After replacing $(G, X)$ by a pair $\left(G_{*}, X_{*}\right)$ and applying $(7.1)$, we may assume that $T_{x}$ splits over a quadratic extension of $F$. On applying (9.1) and (9.3) to the automorphic vector bundle $\mathscr{V}$ defined by the adjoint representation, we find that the map $r_{x}(\mathrm{~g}): G(\mathbb{Q}) \rightarrow G L(\mathrm{~g})$ is defined by an algebraic map $\rho$. Since we know the theorem for each $H_{\alpha}$, the restriction of $\rho$ to the algebraic subgroup $H_{\alpha}$ of $G$ is given be the adjoint representation, and the next lemma shows that this implies that $\rho$ itself is given by the adjoint representation.

Lemma 9.5. The group $G$ is generated (as an algebraic group) by the subgroups $H_{\alpha}$.

Proof. This follows from the fact that, for any embedding $\sigma$ of $F$ into $\mathbb{R},\left[\mathfrak{p}_{\sigma}, \mathfrak{p}_{\sigma}\right]$ $=\mathfrak{l}_{\sigma}$, where $\mathfrak{g}_{\sigma}=\mathfrak{l}_{\sigma} \oplus \mathfrak{p}_{\sigma}$ is a Cartan decomposition of $\mathfrak{g}_{\sigma} \stackrel{d f}{=} \operatorname{Lie}\left(G \otimes_{F, \sigma} \mathbb{R}\right)$.

This completes the second proof of Theorem 3.10.

\section{Appendix : Pairs defining connected and nonconnected Shimura varieties}

In this section, we review some results that allow one to modify the centres of the groups defining Shimura varieties. Throughout, $t$ denotes complex conjugation.

Lemma A.1. Let $G$ be a semisimple adjoint group over a field of characteristic zero, and let $L$ be a finite Galois extension of $k$ that splits $G$. For any finite central covering $G^{\prime} \rightarrow G$ of $G$, there exists a central extension defined over $k$

$$
1 \rightarrow N \rightarrow G_{1} \rightarrow G \rightarrow 1
$$

such that $G_{1}^{\text {der }}=G^{\prime}$ and $N$ is equal to a product of copies of $\operatorname{Res}_{L / k} \mathbb{G}_{m}$.

Proof. See for example Milne and Shih (1982b), 3.1. 
Proposition A.2. For any pair $(G, X)$ defining a connected Shimura variety, there exists a pair $\left(G_{1}, X_{1}\right)$ defining a Shimura variety and such that

(a) $\left(G_{1}, X_{1}\right)^{+}=(G, X)$;

(b) the weight $w_{X}$ of any $h \in X_{1}$ is defined over $\mathbf{Q}$; Q.

(c) the centre $Z\left(G_{1}\right)$ of $G_{1}$ is a product of copies of $\operatorname{Res}_{L \mathbb{Q}} \mathbb{G}_{m}$ for some CM-field $L$ Galois over

Proof. Let $x$ be a special point of $X$, and let $T$ be a maximal torus of $G^{\text {ad }}$ such that $h$ factors through $T_{\mathbb{R}}$. Then $T_{R}$ is anisotropic, and so $\imath$ acts as -1 on $X^{*}(T)$. It follows that, for any $\tau \in$ Aut $(\mathbb{C})$, $\tau l$ and $\imath \tau$ have the same action on $X^{*}(T)$, and so $T$ splits over a $C M$-field $L$, which can be chosen to be Galois over $\mathbb{Q}$. Construct $G_{1}$ as in the lemma with $G_{1}^{\text {der }}=G$ and $G_{1} / N=G^{\text {ad }}$. The inverse image $T_{1}$ of $T$ in $G_{1}$ is a maximal torus. Choose $\mu_{1} \in X_{*}\left(T_{1}\right)$ to lift $\mu \in X_{*}(T)$. The weight $w_{1} \stackrel{d f}{=}-\mu_{1}-l \mu_{1}$ of $\mu_{1}$ lies in $X_{*}\left(Z_{1}\right)$, where $Z_{1}=Z\left(G_{1}\right)=N$. Clearly $\imath w_{1}=w_{1}$ and so, as $H^{1}\left(\mathbb{R}, X_{*}\left(Z_{1}\right)\right)=0$, there exists a $\mu_{0} \in X_{*}\left(Z_{1}\right)$ such that $(t+1) \mu_{0}=w_{1}$. When we replace $\mu_{1}$ by $\mu_{1}-\mu_{0}$ we find that $w_{1}=0$; in particular, $w_{1}$ is defined over $\mathbb{Q}$. Let $h_{1}: \mathbf{S} \rightarrow G_{1}$ correspond to $\mu_{1}$, and let $X_{1}$ be the conjugacy class of $h_{1}$. Then $\left(G_{1}, X_{1}\right)$ fulfills the requirements.

Now let $(G, X)$ be a pair defining a Shimura variety. Recall that $x \in X$ is special if $h_{x}$ factors

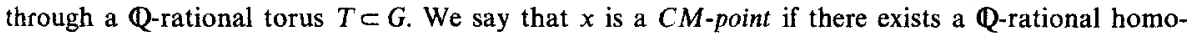
morphism $\rho_{x}: \subseteq \rightarrow G$ such that $\mu_{\mathrm{can}}{ }^{\circ} \rho_{x}=\mu_{x}$. Clearly a $C M$-point is special, and a special point is $C M$ if and only if

$$
(\tau-1)(l+1) \mu_{x}=0=(l+1)(\tau-1) \mu_{x}
$$

all $t \in \operatorname{Aut}(\mathbb{C})$. (Note, pace Deligne (1979), 2.2.4, it is important to distinguish these notions.)

Proposition A.3. Every special point of $X$ is a CM-point when $(G, X)$ satisfies the conditions:

(a) the weight $w_{X}$ is defined over $\mathbb{Q}$;

(b) the centre $Z$ of $G$ is split by a CM-field.

Proof. The conditions say respectively that

$$
\begin{aligned}
& (\tau-1)(\imath+1) \mu_{x}=0, \quad \text { for all } \tau \in \operatorname{Aut}(\mathbb{C}), \quad x \in X ; \\
& \tau \imath \mu=\imath \tau \mu, \quad \text { for all } \tau \in \operatorname{Aut}(\mathbb{C}), \quad \mu \in X_{*}(Z) .
\end{aligned}
$$

Let $x \in X$ be a special, and let $T$ be a maximal torus through which $h_{x}$ factors. The argument in the proof of (A.2) shows that $\tau \imath \mu=\imath \tau \mu$ for $\mu \in X_{*}(T / Z)$, and since

$$
X_{*}(T) \otimes \mathbb{Q}=X_{*}(Z) \otimes \mathbb{Q} \oplus X_{*}(T / Z) \otimes \mathbb{Q}
$$

we see that the same equation holds for $\mu \in X_{*}(T)$. Therefore $(l+1)(\tau-1) \mu_{x}=(\tau-1)(t+1) \mu_{x}$, and we have already observed that this is zero.

Corollary A.4. For any pair $(G, X)$ defining a connected Shimura variety, there exists a pair $\left(G_{1}, X_{1}\right)$ defining a Shimura variety and such that
(a) $\left(G_{1}, X_{1}\right)^{+}=(G, X)$;
(b) every special point of $X_{1}$ is $C M$;
(c) $H^{1}\left(k, Z\left(G_{1}\right)\right)=0$ for all fields $k$ of characteristic zero.

Proof. Combine the last two results.

Proposition A.5. Assume that $(G, X)$ satisfies the conditions of (A.3). There then exists a Q-rational reductive group $G_{0} \subset G$ such that

(a) all $h \in X$ factor through $G_{0 \mathbf{R}}$, and

(b) ad $h(\mathrm{i})$ is a Cartan involution on $G_{0} / w_{X}\left(\mathbb{G}_{m}\right)$.

Proof. We assume that no proper $Q$-rational reductive subgroup of $G$ satisfies (a) and show that $G$ then satisfies (b). Let $H=G /\left(G^{\text {der }} \cdot w\left(\mathbb{G}_{m}\right)\right)$. Then $X^{*}\left(Z / w\left(\mathbb{G}_{m}\right)\right) \otimes \mathbb{Q}=X^{*}(H) \otimes \mathbb{Q}$, and so (A.3b) implies that $\imath \tau=\tau \imath$ on $X^{*}(H)$ for all $\tau \in \operatorname{Aut}(\mathbb{C})$. Let $V^{+}$be the maximal subspace of $X^{*}(H) \otimes \mathbb{Q}$ on which $\imath$ acts as +1 . The last statement shows that $V^{+}$is stable under Aut $(\mathbb{C})$. There therefore 
exists a quotient torus $H^{+}$of $H$ such that $V^{+}=X^{*}\left(H^{+}\right) \otimes \mathbb{Q}$. For any $x \in X, \mathbf{S} / \mathbb{G}_{m} \stackrel{h_{x}}{\longrightarrow} G_{\mathbf{R}} / w\left(\mathbb{G}_{m}\right) \rightarrow H_{\mathbf{R}}^{+}$ is trivial because $\imath$ acts as -1 on $X^{*}\left(\mathbb{S} / \mathbb{G}_{m}\right)$, and so $h_{x}$ factors through $\operatorname{Ker}\left(G_{\mathbf{R}} \rightarrow H_{\mathbf{R}}^{+}\right)$; we must have $H^{+}=0$. It follows now that $\left(Z / w\left(\mathbb{G}_{m}\right)\right)_{R}$ is anisotropic, and (b) holds.

Remark A.6. For the group $G_{0}$ constructed in (A.4), $Z\left(G_{0}\right)(\mathbb{Q})$ is discrete in $Z\left(G_{0}\right)\left(\mathbb{A}^{\mathcal{S}}\right)(\mathrm{cf}$. Deligne (1979), 2.1.11).

\section{Bibliography}

Borovoi, M.: Langlands's conjecture concerning conjugation of Shimura varieties. Sel. Math. Sov. 3, 3-39 (1983/84)

Deligne, P.: Equations Différentielles à Points Singuliers Réguliers. (Lect. Notes Math., vol. 163) Berlin Heidelberg New York: Springer 1970

Deligne, P.: Travaux de Shimura. Sém. Bourbaki, Fév. 1971, Exposé 389, Lect. Notes Math. vol. 244. Berlin Heidelberg New York: Springer 1971

Deligne, $P$.: Variétiés de Shimura: interpretation modulaire, et techniques de construction de modéles canoniques. Proc. Symp. Pure Math., A.M.S., 33, part 2, 247-290 (1979)

Deligne, P.: Hodge cycles on abelian varieties (Notes by J.S. Milne). In: Hodge Cycles, Motives, and Shimura Varieties (Lect. Notes Math., vol. 900, Berlin Heidelberg New York: Springer 1982a, pp. 9-100)

Deligne, P.: Motifs et groupes de Taniyama. In: Hodge Cycles, Motives, and Shimura Varieties, (Lect. Notes Math., vol. 900) Berlin Heidelberg New York: Springer 1982b, pp. 261-279

Deligne, P., Milne, J.: Tannakian categories. In: Hodge Cycles, Motives, and Shimura Varieties. (Lect. Notes Math., vol. 900) Berlin Heidelberg New York: Springer 1982, pp. 101-228

Grothendieck, A.: Etude locale des schémes et des morphismes de schémas. Inst. Hautes Etudes Sci., Publ. Math. 32, 5-361 (1967)

Harris, M.: Arithmetic vector bundles on Shimura varieties. In: (Satake I., Morita, Y. (ed).) Automorphic Forms of Several Variables Prog. Math. 46, Birkhäuser, Boston, 1984, pp. 138-159

Harris, M.: Arithmetic vector bundles and automorphic forms on Shimura varieties. I. Invent. math. 82, 151-189 (1985)

Hartshorne, R.: Ample Subvarieties of Algebraic Varieties. Lect. Notes Math., vol. 156) Berlin Heidelberg New York: Springer 1970

Helgason, S.: Differential Geometry, Lie Groups, and Symmetric Spaces. New York: Academic Press, 1978

Kobayashi, S., Nomizu, K.: Foundations of Differential Geometry I, II, New York: Wiley (Interscience) $1963 / 69$

Langlands, R.: Automorphic representations, Shimura varieties, and motives. Ein Märchen. Proc. Symp. Pure Math., A.M.S., 33, 205-246 (1979)

Milne, J.: Etale Cohomology, Princeton; Princeton University Press 1980

Milne, J.: The action of an automorphism of $\mathbb{C}$ on a Shimura variety and its special points. Prog. Math. Vol. 35, Boston: Birkhäuser, 1983, pp. 239--265

Milne, J.: Arithmetic Duality Theorems, Perspectives in Math., 1, Boston: Academic Press 1986

Milne, J., Shih, K.-y.: Langlands's construction of the Taniyama group. In: Hodge Cycles, Motives, and Shimura Varieties (Lect. Notes Math., vol. 900, Berlin Heidelberg New York: Springer 1982a, pp. 229-260

Milne, J., Shih, K.-y.: Conjugates of Shimura varieties. In: Hodge Cycles, Motives, and Shimura Varieties (Lect. Notes Math., vol. 900, Berlin Heidelberg New York: Springer 1982 b, pp. 280-356

Mumford, D.: Geometric Invariant Theory. Berlin Heidelberg New York: Springer 1965

Murakami, S.: Cohomology Groups of Vector-valued Forms on Symmetric Spaces, mimeographed notes, University of Chicago, 1966

Platonov, V., Rapincuk, A.: On the group of rational points of three-dimensional groups. Sov. Math. Dokl. 20, 693-697 (1979)

Saavedra Rivano, N.: Catégories Tannakiennes. (Lect. Notes Math., vol. 265) Berlin Heidelberg New York: Springer 1972

Shimura, G.: On some problems of algebraicity. Proc. Int. Cong. Math. 1978, Vol I, pp. 373-379 